Geometry \& Topology

Volume 8 (2004) 35-76

Published: 21 January 2004

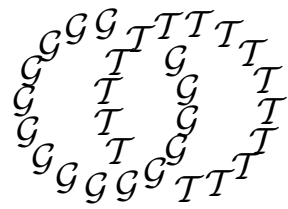

\title{
Rohlin's invariant and gauge theory II. Mapping tori
}

\author{
DANIEL RUBERMAN \\ Nikolai SaVELIEV \\ Department of Mathematics, MS 050, Brandeis University \\ Waltham, MA 02454, USA \\ and \\ Department of Mathematics, University of Miami \\ PO Box 249085, Coral Gables, FL 33124, USA \\ Email: ruberman@brandeis.edu and saveliev@math.miami.edu
}

\begin{abstract}
This is the second in a series of papers studying the relationship between Rohlin's theorem and gauge theory. We discuss an invariant of a homology $S^{1} \times S^{3}$ defined by Furuta and Ohta as an analogue of Casson's invariant for homology 3-spheres. Our main result is a calculation of the Furuta-Ohta invariant for the mapping torus of a finite-order diffeomorphism of a homology sphere. The answer is the equivariant Casson invariant (Collin-Saveliev 2001) if the action has fixed points, and a version of the Boyer-Nicas (1990) invariant if the action is free. We deduce, for finite-order mapping tori, the conjecture of Furuta and Ohta that their invariant reduces mod 2 to the Rohlin invariant of a manifold carrying a generator of the third homology group. Under some transversality assumptions, we show that the Furuta-Ohta invariant coincides with the Lefschetz number of the action on Floer homology. Comparing our two answers yields an example of a diffeomorphism acting trivially on the representation variety but non-trivially on Floer homology.
\end{abstract}

AMS Classification numbers Primary: 57R57

Secondary: 57R58

Keywords: Casson invariant, Rohlin invariant, Floer homology

Proposed: Ronald Stern

Received: 30 June 2003

Seconded: Robion Kirby, Tomasz Mrowka

Revised: 18 December 2003

(c) Geometry ${ }^{\mathcal{S}} \mathcal{T}$ Topology Publications 


\section{Introduction}

Let $X$ be a $\mathbb{Z}[\mathbb{Z}]$-homology $S^{1} \times S^{3}$, that is, a smooth closed oriented 4manifold such that $H_{*}(X ; \mathbb{Z})=H_{*}\left(S^{1} \times S^{3} ; \mathbb{Z}\right)$ and $H_{*}(\tilde{X} ; \mathbb{Z})=H_{*}\left(S^{3} ; \mathbb{Z}\right)$, where $\tilde{X}$ is the universal abelian cover of $X$. Denote by $\mathcal{M}^{*}(X)$ the moduli space of irreducible ASD connections on a trivial $S U(2)$-bundle over $X$. The virtual dimension of $\mathcal{M}^{*}(X)$ is $-3\left(1-b_{1}+b_{2}^{+}\right)(X)=0$. In fact, $\mathcal{M}^{*}(X)$ coincides with the moduli space of irreducible flat connections on $X$. According to [8] and [13], the space $\mathcal{M}^{*}(X)$ is compact and canonically oriented once an orientation on $H^{1}(X ; \mathbb{R})=\mathbb{R}$ is fixed. After a perturbation if necessary, it is a finite collection of non-degenerate points. A signed count of these points is an invariant of $X$ known as the Donaldson polynomial $D_{0}(X)$ of degree zero. Furuta and Ohta 13 define1 $\lambda_{F O}(X)=D_{0}(X) / 4$. We will refer to $\lambda_{F O}(X)$ as the Furuta-Ohta invariant.

The Furuta-Ohta invariant may be regarded as a 4-dimensional version of Casson's invariant. Associated to $X$, there is also a Rohlin-type invariant, defined as the usual Rohlin invariant of a 3 -manifold carrying a generator of the third homology. (The definition of this invariant given in [13] is somewhat different from the one we use, which comes from [23], but the two definitions can be readily shown to agree.) Furuta and Ohta [13] conjectured that the modulo 2 reduction of $\lambda_{F O}(X)$ equals the Rohlin invariant of $X$, by analogy with Casson's result that his invariant gives the Rohlin invariant of a homology 3 -sphere.

The goal of this paper is to calculate the Furuta-Ohta invariant in the special case when

$$
X=[0,1] \times \Sigma /(0, x) \sim(1, \tau(x))
$$

is the mapping torus of a finite order orientation preserving diffeomorphism $\tau: \Sigma \rightarrow \Sigma$ of an integral homology sphere $\Sigma$. The mapping torus $X$ is a smooth 4 -manifold oriented by the volume form $d t \wedge \operatorname{vol}_{\Sigma}$. It is obviously a $\mathbb{Z}[\mathbb{Z}]$-homology $S^{1} \times S^{3}$. We show that the Furuta-Ohta invariant of $X$ is the equivariant Casson invariant of the pair $(\Sigma, \tau)$.

More precisely, let $\mathcal{R}^{*}(\Sigma)$ be the space of irreducible flat connections in the bundle $\Sigma \times S U(2)$ modulo gauge equivalence, and let $\tau^{*}: \mathcal{R}^{*}(\Sigma) \rightarrow \mathcal{R}^{*}(\Sigma)$ be the map induced by pulling back connections. The fixed point set of $\tau^{*}$ will be denoted by $\mathcal{R}^{\tau}(\Sigma)$. After perturbation if necessary, the space $\mathcal{R}^{\tau}(\Sigma)$ consists of

\footnotetext{
${ }^{1}$ Actually they use $D_{0}(X) / 2$; we divide by 4 to make the definition compatible with their conjecture, stated in the next paragraph.
} 
finitely many non-degenerate points which can be counted with signs to obtain the equivariant Casson invariant $\lambda^{\tau}(\Sigma)$. A rigorous definition of $\lambda^{\tau}(\Sigma)$ for $\tau$ having fixed points can be found in [7. The definition of $\lambda^{\tau}(\Sigma)$ for a fixed point free $\tau$ is given in this paper; it is related to the Boyer-Nicas invariant [3, 4] of $\Sigma / \tau$. Note that, if $\tau=$ id, the equivariant Casson invariant coincides with the regular Casson invariant $\lambda(\Sigma)$.

Theorem 1.1 Let $\Sigma$ be an integral homology sphere and let $\tau: \Sigma \rightarrow \Sigma$ be an orientation preserving diffeomorphism of finite order. If $X$ is the mapping torus of $\tau$ then

$$
\lambda_{F O}(X)=\lambda^{\tau}(\Sigma)
$$

We further show how to express $\lambda^{\tau}(\Sigma)$ in terms of the regular Casson invariant and certain classical knot invariants, compare with [7. Once such an explicit formula is in place, we will prove in Section 8 the following result, verifying the conjecture of Furuta and Ohta for the mapping tori of finite order diffeomorphisms.

Theorem 1.2 Let $\tau: \Sigma \rightarrow \Sigma$ be an orientation preserving diffeomorphism of finite order then the modulo 2 reduction of $\lambda^{\tau}(\Sigma)$ equals the Rohlin invariant of $\Sigma$.

An alternate approach to the invariant $\lambda_{F O}$ of a mapping torus is via the 'TQFT' view of Donaldson-Floer theory [9]. The diffeomorphism $\tau$ induces an automorphism on the instanton Floer homology of $\Sigma$. Under the assumption that the representation variety $\mathcal{R}^{*}(\Sigma)$ is non-degenerate, we prove in Theorem 3.8 that the Furuta-Ohta invariant of $X$ equals half the Lefschetz number of this automorphism. We conjecture that this is the case in general, but the lack of equivariant perturbations is a non-trivial obstacle to the proof. A comparison of this result with Theorem 1.1 gives rise to the surprising phenomenon that a map $\tau$ may act by the identity on $\mathcal{R}^{*}(\Sigma)$, but still act non-trivially on the Floer homology. Examples are given in Section 9 ,

The basic idea behind Theorem 1.1 is that for mapping tori, $\lambda_{F O}$ counts fixed points of $\tau^{*}$, with multiplicity 2 coming from the choice of holonomy in the circle direction. Of course, this must properly take account of the signs with which flat connections are counted, and of the perturbations used in the two theories.

The authors are grateful to Fred Diamond, Chris Herald, Chuck Livingston, Tom Mrowka, and Liviu Nicolaescu for useful remarks and for sharing their expertise. The first author was partially supported by NSF Grants 9971802 and 0204386. The second author was partially supported by NSF Grant 0305946. 


\section{Some equivariant gauge theory}

Let $\mathcal{A}(\Sigma)$ be the affine space of connections in a trivialized $S U(2)$-bundle $P$ over $\Sigma$, and let $\mathcal{A}^{*}(\Sigma)$ be the subset consisting of irreducible connections. Any endomorphism $\tilde{\tau}: P \rightarrow P$ which lifts $\tau: \Sigma \rightarrow \Sigma$ induces an action on connections by pull back. For any two lifts, $\tilde{\tau}_{1}$ and $\tilde{\tau}_{2}$, there obviously exists a gauge transformation $g: P \rightarrow P$ such that $\tilde{\tau}_{2}=\tilde{\tau}_{1} \cdot g$. This observation shows that we have a well defined action $\tau^{*}$ on $\mathcal{B}^{*}(\Sigma)=\mathcal{A}^{*}(\Sigma) / \mathcal{G}(\Sigma)$. The fixed point set of $\tau^{*}$ will be called $\mathcal{B}^{\tau}(\Sigma)$.

\subsection{Decomposing $\mathcal{B}^{\tau}(\Sigma)$}

Let $\alpha$ be a connection on $P$ whose gauge equivalence class belongs to $\mathcal{B}^{\tau}(\Sigma)$. Then there is a lift $\tilde{\tau}: P \rightarrow P$ such that $\tilde{\tau}^{*} \alpha=\alpha$. Since $\alpha$ is irreducible, the lift $\tilde{\tau}$ is defined uniquely up to a sign. Moreover, $\tilde{\tau}^{n}$ is an endomorphism of $P$ lifting the identity map hence $\left(\tilde{\tau}^{n}\right)^{*} \alpha=\alpha$ implies that $\tilde{\tau}^{n}= \pm 1$. This allows one to decompose $\mathcal{B}^{\tau}(\Sigma)$ into a disjoint union [6, 31]

$$
\mathcal{B}^{\tau}(\Sigma)=\bigsqcup_{[\tilde{\tau}]} \mathcal{B}^{\tilde{\tau}}(\Sigma)
$$

where the $\tilde{\tau}$ are lifts of $\tau$ such that $\tilde{\tau}^{n}= \pm 1$. The equivalence relation among the lifts $\tilde{\tau}$ is that $\tilde{\tau}_{1} \sim \tilde{\tau}_{2}$ if and only if $\tilde{\tau}_{2}= \pm g \cdot \tilde{\tau}_{1} \cdot g^{-1}$ for some gauge transformation $g: P \rightarrow P$.

The spaces $\mathcal{B}^{\tilde{\tau}}(\Sigma)$ can be described as follows. For a fixed lift $\tilde{\tau}$, let $\mathcal{A}^{\tilde{\tau}}(\Sigma)$ be the subset of $\mathcal{A}^{*}(\Sigma)$ consisting of irreducible connections $A$ such that $\tilde{\tau}^{*} A=A$. Define $\mathcal{G}^{\tilde{\tau}}(\Sigma)=\{g \in \mathcal{G}(\Sigma) \mid g \tilde{\tau}= \pm \tilde{\tau} g\}$ then $\mathcal{B}^{\tilde{\tau}}(\Sigma)=\mathcal{A}^{\tilde{\tau}}(\Sigma) / \mathcal{G}^{\tilde{\tau}}(\Sigma)$.

There is also a well defined action $\tau^{*}$ on the flat moduli space $\mathcal{R}^{*}(\Sigma)$ whose fixed point set is $\mathcal{R}^{\tau}(\Sigma)=\mathcal{B}^{\tau}(\Sigma) \cap \mathcal{R}^{*}(\Sigma)$. It splits as

$$
\mathcal{R}^{\tau}(\Sigma)=\bigsqcup_{[\tilde{\tau}]} \mathcal{R}^{\tilde{\tau}}(\Sigma)
$$

We wish to ramify the above splittings for later use. Note that any lift $\tilde{\tau}: P \rightarrow$ $P$ can be written in the base-fiber coordinates as $\tilde{\tau}(x, y)=(\tau(x), \rho(x) y)$ for some $\rho: \Sigma \rightarrow S U(2)$. The lift $\tilde{\tau}$ is said to be constant if there exists $u \in S U(2)$ such that $\rho(x)=u$ for all $x \in \Sigma$. The rest of this section is devoted to proving the following result.

Proposition 2.1 There are finitely many equivalence classes $[\tilde{\tau}]$ in the decomposition (11), and each of them contains a constant lift. 


\subsection{The case of non-empty $\operatorname{Fix}(\tau)$}

Let us first suppose that $\operatorname{Fix}(\tau)$ is non-empty. Then $\Sigma / \tau$ is an integral homology sphere and the projection map $\Sigma \rightarrow \Sigma / \tau$ is a branched covering with branch set a knot. Let $\tilde{\tau}(x, y)=(\tau(x), \rho(x) y)$ for some $\rho: \Sigma \rightarrow S U(2)$. If $x \in \operatorname{Fix}(\tau)$ then $\tilde{\tau}(x, y)=(x, \rho(x) y)$ and $\tilde{\tau}^{n}(x, y)=\left(x, \rho(x)^{n} y\right)=(x, \pm y)$. This implies that $\rho(x)^{n}= \pm 1$ and, in particular, that $\operatorname{tr} \rho(x)$ can only take finitely many distinct values. Since $\operatorname{Fix}(\tau)$ is connected, $\rho(\operatorname{Fix}(\tau))$ has to belong to a single conjugacy class in $S U(2)$. Replacing $\tilde{\tau}$ by an equivalent lift if necessary, we may assume that $\rho(x)=u$ for all $x \in \operatorname{Fix}(\tau)$.

Let $u: P \rightarrow P$ be the constant lift $u(x, y)=(\tau(x), u \cdot y)$ and consider the $S O(3)$ orbifold bundles $P / \tilde{\tau}$ and $P / u$ over $\Sigma / \tau$. All such bundles are classified by the holonomy around the singular set in $\Sigma / \tau$. Since this holonomy equals $\operatorname{ad}(u)$ in both cases, the bundles $P / \tilde{\tau}$ and $P / u$ are isomorphic. Take any isomorphism and pull it back to a gauge transformation $g: P \rightarrow P$. It is clear that $\tilde{\tau}= \pm g \cdot u \cdot g^{-1}$ and hence $\mathcal{B}^{\tilde{\tau}}(\Sigma)=\mathcal{B}^{u}(\Sigma)$. Thus (1) becomes a finite decomposition

$$
\mathcal{B}^{\tau}(\Sigma)=\bigsqcup_{|\operatorname{tr} u|} \mathcal{B}^{u}(\Sigma)
$$

\subsection{The case of empty $\operatorname{Fix}(\tau)$}

Now suppose that $\operatorname{Fix}(\tau)$ is empty. Then $\Sigma / \tilde{\tau}$ is a homology lens space and the projection map $\Sigma \rightarrow \Sigma / \tilde{\tau}$ is a regular (unbranched) covering. The bundle $P$ gives rise to the $S O(3)$-bundle $P / \tilde{\tau}$ on $\Sigma / \tilde{\tau}$. Such bundles are classified by their Stiefel-Whitney class $w_{2}(P / \tilde{\tau})$ so there are two different bundles if $n$ is even, and just one if $n$ is odd.

Note that the trivial bundle can be realized as the quotient bundle of the constant lift $u(x, y)=(\tau(x), y)$, and the non-trivial bundle (in the case of even $n$ ) as the quotient bundle of the constant lift $u(x, y)=(\tau(x), u \cdot y)$ where $u$ is any matrix in $S U(2)$ such that $u^{n}=-1$. Any isomorphism between $P / \tilde{\tau}$ and $P / u$, for either of the above lifts $u$, pulls back to a gauge transformation $g: P \rightarrow P$ such that $\tilde{\tau}= \pm g \cdot u \cdot g^{-1}$. Therefore, the equivalence classes of lifts $\tilde{\tau}$ are classified by $w_{2}(P / \tilde{\tau})$. 


\section{The unperturbed case}

In this section, we prove Theorem [1.1 under the assumption that $\mathcal{R}^{\tau}(\Sigma)$ is non-degenerate, leaving the degenerate case to later sections. We first establish a two-to-one correspondence between the flat moduli spaces $\mathcal{M}^{*}(X)$ and $\mathcal{R}^{\tau}(\Sigma)$, then compare the non-degeneracy conditions in the two settings. Finally, the orientations of $\mathcal{M}^{*}(X)$ and $\mathcal{R}^{\tau}(\Sigma)$ are compared using the concept of orientation transport, see 22 .

\subsection{Identifying flat moduli spaces}

Let $X$ be the mapping torus of $\tau: \Sigma \rightarrow \Sigma$. Denote by $\mathcal{B}^{*}(X)=\mathcal{A}^{*}(X) / \mathcal{G}(X)$ the moduli space of irreducible connections in a trivial $S U(2)$ bundle on $X$, and by $\mathcal{M}^{*}(X) \subset \mathcal{B}^{*}(X)$ the respective anti-self-dual moduli space. Since the bundle is trivial, a standard Chern-Weil argument implies that $\mathcal{M}^{*}(X)$ consists of flat connections. Let $i: \Sigma \rightarrow X$ be the embedding $i(x)=[0, x]$.

Proposition 3.1 The map $i^{*}: \mathcal{M}^{*}(X) \rightarrow \mathcal{R}^{\tau}(\Sigma)$ induced by pulling back connections is a well defined two-to-one correspondence.

Proof First note that, for any irreducible flat connection $A$ on $X$, its pull back $i^{*} A$ is also irreducible: if $i^{*} A$ were reducible it would have to be trivial which would obviously contradict the irreducibility of $A$.

Let $P$ be a trivialized $S U(2)$-bundle over $\Sigma$. Given a flat connection $A$ over $X$, cut $X$ open along $i(\Sigma)$ and put $A$ into temporal gauge over $[0,1] \times \Sigma$. We obtain a path $A(t)$ of connections in $P$. Note that $A(0)$ and $A(1)$ need not be equal but they certainly are gauge equivalent (via the holonomy along the intervals $[0,1] \times\{x\})$. Thus $A(1)=\tilde{\tau}^{*} A(0)$ for some bundle automorphism $\tilde{\tau}: P \rightarrow P$ lifting $\tau$. In temporal gauge, the flatness equation $F(A)=0$ takes the form $d t \wedge A^{\prime}(t)+F(A(t))=0$ hence $A(t)=\alpha$ is a constant path, where $\alpha$ is a flat connection over $\Sigma$. Since $\tilde{\tau}^{*} A(0)=A(1)$ we conclude that $\tilde{\tau}^{*} \alpha=\alpha$.

Note that, conversely, $A$ can be obtained from $\alpha$ by pulling $\alpha$ back to $[0,1] \times \Sigma$ and identifying the ends via $\tilde{\tau}$.

Now, we need to see how the above correspondence behaves with respect to gauge transformations. Let us fix a lift $\tilde{\tau}$. Suppose that $A$ and $A^{\prime}$ are connections that are in temporal gauge when pulled back to $[0,1] \times \Sigma$, and that they are equivalent via a gauge transformation $g$. It is straightforward to show 
that the restriction of $g \in \mathcal{G}(X)$ to $[0,1] \times \Sigma$ must be constant in $t$. Since $g$ defines a gauge transformation over $X$, it also satisfies the boundary condition $\tilde{\tau} g=g \tilde{\tau}$. This identifies $\mathcal{M}^{*}(X)$ with the space of irreducible flat connections in $P$ modulo the index two subgroup of $\mathcal{G}^{\tilde{\tau}}(\Sigma)$ which consists of gauge transformations $g: P \rightarrow P$ such that $\tilde{\tau} g=g \tilde{\tau}$. Because of the irreducibility, this leads to a two-to-one correspondence between $\mathcal{M}^{*}(X)$ and $\mathcal{R}^{\tilde{\tau}}(\Sigma)$.

Remark 3.2 Note that the construction of a connection over $X$ via pulling an equivariant connection $\alpha$ back to $[0,1] \times \Sigma$ and then identifying the ends makes sense for all connections $\alpha \in \mathcal{A}^{\tilde{\tau}}(\Sigma)$ and not just the flat ones. We will denote the respective map by $\pi: \mathcal{A}^{\tilde{\tau}}(\Sigma) \rightarrow \mathcal{A}(X)$.

\subsection{The non-degeneracy condition}

The moduli space $\mathcal{R}^{\tau}(\Sigma)$ is called non-degenerate if the equivariant cohomology groups $H_{\tau}^{1}(\Sigma$; ad $\alpha)$ vanish for all $\alpha \in \mathcal{R}^{\tau}(\Sigma)$, compare with [7]. The moduli space $\mathcal{M}^{*}(X)$ is called non-degenerate if $\operatorname{coker}\left(d_{A}^{*} \oplus d_{A}^{+}\right)=0$ for all $A \in \mathcal{M}^{*}(X)$.

Proposition 3.3 The moduli space $\mathcal{M}^{*}(X)$ is non-degenerate if and only if $\mathcal{R}^{\tau}(\Sigma)$ is non-degenerate.

Proof Since the formal dimension of $\mathcal{M}^{*}(X)$ is zero, $\operatorname{ind}\left(d_{A}^{*} \oplus d_{A}^{+}\right)=0$, and proving that $\operatorname{coker}\left(d_{A}^{*} \oplus d_{A}^{+}\right)=0$ is equivalent to proving that $\operatorname{ker}\left(d_{A}^{*} \oplus d_{A}^{+}\right)=0$. The connection $A$ is flat and therefore the latter is equivalent to showing that $H^{1}(X ; \operatorname{ad} A)=0$. The group $H^{1}(X ;$ ad $A)$ can be computed with the help of the Leray-Serre spectral sequence applied to the fibration $X \rightarrow S^{1}$ with fiber $\Sigma$. The $E_{2}$-term of this spectral sequence is

$$
E_{2}^{p q}=H^{p}\left(S^{1}, \mathcal{H}^{q}(\Sigma ; \operatorname{ad} \alpha)\right),
$$

where $\alpha=i^{*} A$ and $\mathcal{H}^{q}(\Sigma$; ad $\alpha)$ is the local coefficient system associated with the fibration. The groups $E_{2}^{p q}$ vanish for all $p \geq 2$ hence the spectral sequence collapses at the second term, and

$$
H^{1}(X ; \operatorname{ad} A)=H^{1}\left(S^{1}, \mathcal{H}^{0}(\Sigma ; \operatorname{ad} \alpha)\right) \oplus H^{0}\left(S^{1}, \mathcal{H}^{1}(\Sigma ; \operatorname{ad} \alpha)\right) .
$$

Since $\alpha$ is irreducible, $H^{0}(\Sigma ; \operatorname{ad} \alpha)=0$ and the first summand in the above formula vanishes. The generator of $\pi_{1}\left(S^{1}\right)$ acts on $H^{1}(\Sigma ; \operatorname{ad} \alpha)$ as $\tau^{*}: H^{1}(\Sigma ; \operatorname{ad} \alpha)$ $\rightarrow H^{1}(\Sigma ; \operatorname{ad} \alpha)$, therefore, $H^{0}\left(S^{1}, \mathcal{H}^{1}(\Sigma ; \operatorname{ad} \alpha)\right)$ is the fixed point set of $\tau^{*}$, which is the equivariant cohomology $H_{\tau}^{1}(\Sigma ; \operatorname{ad} \alpha)$. Thus we conclude that $H^{1}(X ; \operatorname{ad} A)=H_{\tau}^{1}(\Sigma ; \operatorname{ad} \alpha)$, which completes the proof. 


\subsection{Orientation transport}

Let $M$ be a smooth closed oriented Riemannian manifold and let $D: C^{\infty}(\xi) \rightarrow$ $C^{\infty}(\eta)$ be a first order elliptic operator such that ind $D=0$. Given a smooth family $h$ of bundle isomorphisms $h_{s}: \xi \rightarrow \eta, 0 \leq s \leq 1$, referred to as homotopy, form a family of elliptic operators $D_{s}: C^{\infty}(\xi) \rightarrow C^{\infty}(\eta)$ by the rule $D_{s}=D+h_{s}$. All these operators have the same symbol; in particular, ind $D_{s}=0$ for all $s \in[0,1]$.

Let us fix orientations on the lines $\operatorname{det} D_{i}=\operatorname{det}\left(\operatorname{ker} D_{i} \otimes\left(\operatorname{coker} D_{i}\right)^{*}\right), i=0,1$. The homotopy $h$ provides an isomorphism $\psi$ : $\operatorname{det} D_{0} \rightarrow \operatorname{det} D_{1}$. We say that the orientation transport along homotopy $h$ is 1 if $\psi$ is orientation preserving, and -1 otherwise. We use notation $\varepsilon\left(D_{0}, h, D_{1}\right)= \pm 1$ for the orientation transport, to indicate its dependence on the choice of orientations and the homotopy $h$.

Once the orientations on $\operatorname{det} D_{i}, i=0,1$, are fixed, the orientation transport $\varepsilon\left(D_{0}, h, D_{1}\right)$ only depends on the homotopy class of $h$ rel $\{0,1\}$. It is given by the following formula, see [22, page 95]. Define the resonance set of the homotopy $h$ as

$$
\mathcal{Z}_{h}=\left\{s \in[0,1] \mid \operatorname{ker} D_{s} \neq 0\right\} .
$$

For each $s \in[0,1]$ denote by $P_{s}$ the orthogonal projection onto coker $D_{s}$. Let $h_{s}^{\prime}$ be the derivative of the bundle isomorphism $h_{s}: \xi \rightarrow \eta$. The homotopy is called regular if its resonance set is finite and, for any $s \in[0,1]$, the resonance operator

$$
R_{s}=P_{s} \circ h_{s}^{\prime}: \operatorname{ker} D_{s} \longrightarrow L^{2}(\eta) \longrightarrow \operatorname{coker} D_{s}
$$

is a linear isomorphism. Suppose $h$ is a regular homotopy and set $d_{s}=$ $\operatorname{dim} \operatorname{ker} D_{s}=\operatorname{dim} \operatorname{coker} D_{s}$. Then

$$
\varepsilon\left(D_{0}, h, D_{1}\right)=\operatorname{sign} R_{0} \cdot \operatorname{sign} R_{1} \cdot \prod_{s \in[0,1)}(-1)^{d_{s}},
$$

where $\operatorname{sign} R_{i}= \pm 1, i=0,1$, according to whether $\operatorname{det} R_{i} \in \operatorname{det}\left(\operatorname{ker} D_{i} \otimes\right.$ $\left(\operatorname{coker} D_{i}\right)^{*}$ ) is positive or negative.

\subsection{Orientation of $\mathcal{R}^{*}(\Sigma)$}

We assume that $\mathcal{R}^{\tau}(\Sigma)$ is non-degenerate. For any point $\alpha \in \mathcal{R}^{\tau}(\Sigma)$, its orientation is given by

$$
(-1)^{\mathrm{sf}^{\tau}(\theta, \alpha)}
$$


where $\operatorname{sf}^{\tau}(\theta, \alpha)$ is the modulo 2 (equivariant) spectral flow. In fact, equivariant spectral flow is well defined modulo 4 when $\tau \neq$ id and modulo 8 when $\tau=$ id but the modulo 2 spectral flow will suffice for our purposes. The definition is as follows, compare with [7].

Fix a Riemannian metric on $\Sigma$ so that $\tau: \Sigma \rightarrow \Sigma$ is an isometry, and consider a trivialized $S U(2)$-bundle $P$ over $\Sigma$. According to Proposition 2.1, there exists a constant lift $u: P \rightarrow P$ of $\tau$ such that $u^{*} \alpha=\alpha$ and $u^{n}= \pm 1$.

Observe that $u^{*} \theta=\theta$ where $\theta$ is the product connection on $P$, and choose a smooth path $\alpha(s), 0 \leq s \leq 1$, of equivariant connections on $P$ such that $\alpha(0)=\theta$ and $\alpha(1)=\alpha$. The equivariance here means that $u^{*} \alpha(s)=\alpha(s)$ for all $s$; it can be achieved by averaging because connections form an affine space. Associated with $\alpha(s)$ is a path of self-adjoint Fredholm operators $K_{\alpha(s)}^{u}$ obtained by restricting the operators

$$
K_{\alpha(s)}=\left(\begin{array}{cr}
0 & d_{\alpha(s)}^{*} \\
d_{\alpha(s)} & -* d_{\alpha(s)}
\end{array}\right)
$$

onto the space of $u$-equivariant differential forms $\left(\Omega^{0} \oplus \Omega^{1}\right)_{u}(\Sigma$, ad $P)$, where ad $P=P \times_{\text {ad }} \mathfrak{s u}(2)$ is the adjoint bundle of $P$ and $d_{\alpha(s)}$ is the covariant derivative. By $u$-equivariant differential forms $\Omega_{u}^{*}$ we mean the $(+1)$-eigenspace of the pull back operator $u^{*}: \Omega^{*} \rightarrow \Omega^{*}$ induced by the lift $\operatorname{ad}(u): \operatorname{ad} P \rightarrow \operatorname{ad} P$.

The one-parameter family of spectra of operators $K_{\alpha(s)}^{u}$ can be viewed as a collection of spectral curves in the $(s, \lambda)$-plane connecting the spectrum of $K_{\theta}^{u}$ with that of $K_{\alpha}^{u}$. These curves are smooth, at least near zero. The (modulo 2) spectral flow $\mathrm{sf}^{\tau}(\theta, \alpha)$ is the number of eigenvalues, counted with multiplicities, which cross the $s$-axis plus the number of spectral curves which start at zero and go down. An equivalent way to define spectral flow is to consider the straight line connecting the points $(0,-\delta)$ and $(1, \delta)$ where $\delta>0$ is chosen smaller than the absolute value of any non-zero eigenvalue of $K_{\theta}^{u}$ and $K_{\alpha}^{u}$. Then the spectral flow is the number of eigenvalues, counted with multiplicities, which cross this line. The spectral flow is well defined; it only depends on $\alpha$ and not on the choice of $\alpha(s)$.

Proposition 3.4 Let $\alpha \in \mathcal{R}^{\tau}(\Sigma)$ and let $\alpha(s)$ be a path of equivariant connections such that $\alpha(0)=\theta$ and $\alpha(1)=\alpha$. Let $h_{s}=K_{\alpha(s)}^{u}-K_{\theta}^{u}$ then, for any small generic $\delta>0$,

$$
(-1)^{\mathrm{sf}^{\tau}(\theta, \alpha)}=\varepsilon\left(K_{\theta}^{u}+\delta, h, K_{\alpha}^{u}+\delta\right) .
$$


Remark 3.5 If $\delta>0$ is sufficiently small then both kernel and cokernel of $K_{\alpha(s)}^{u}+\delta$ vanish at $s=0$ and $s=1$. This provides canonical orientations for $\operatorname{det}\left(K_{\theta}^{u}+\delta\right)$ and $\operatorname{det}\left(K_{\alpha}^{u}+\delta\right)$, which are implicit in (5).

Proof The resonance set $\mathcal{Z}_{h}$ of $h_{s}$ consists of the values of $s$ at which the spectral curves of $K_{\alpha(s)}^{u}+\delta$ intersect the $s$-axis. These are exactly the points where the spectral curves of $K_{\alpha(s)}^{u}$ intersect the horizontal line $\lambda=-\delta$. According to Sard's theorem, these intersections are transversal for a generic $\delta>0$. Moreover, the modulo 2 count of these intersection points equals $\operatorname{sf}^{\tau}(\theta, \alpha)$ (due to the fact that $\alpha$ is non-degenerate and hence $\left.\operatorname{ker} K_{\alpha}^{u}=0\right)$.

Now suppose that $s_{0} \in \mathcal{Z}_{h}$ and consider a smooth family $\varphi_{s}$ such that

$$
\left(K_{\alpha(s)}^{u}+\delta\right) \varphi_{s}=\lambda_{s} \varphi_{s} \quad \text { and } \quad \lambda_{s_{0}}=0 .
$$

Differentiating with respect to $s$ and setting $s=s_{0}$, we obtain

$$
h_{s_{0}}^{\prime} \varphi_{s_{0}}=\lambda_{s_{0}}^{\prime} \varphi_{s_{0}}-\left(K_{\alpha\left(s_{0}\right)}^{u}+\delta\right) \varphi_{s_{0}}^{\prime} .
$$

The differential form $\left(K_{\alpha\left(s_{0}\right)}^{u}+\delta\right) \varphi_{s_{0}}^{\prime}$ is orthogonal to $\operatorname{coker}\left(K_{\alpha\left(s_{0}\right)}^{u}+\delta\right)$ hence $\varphi_{s_{0}}$ is an eigenform of the resonance operator

$$
R_{s_{0}}: \operatorname{ker}\left(K_{\alpha\left(s_{0}\right)}^{u}+\delta\right) \rightarrow \operatorname{coker}\left(K_{\alpha\left(s_{0}\right)}^{u}+\delta\right)
$$

with eigenvalue $\lambda_{s_{0}}^{\prime}$. The path $\alpha(s)$ was chosen so that $\lambda_{s_{0}}^{\prime} \neq 0$ hence we can conclude that the resonance operator $R_{s_{0}}$ is a linear isomorphism. Since this is true for all $s_{0} \in \mathcal{Z}_{h}$, the homotopy $h_{s}$ is regular. Since $\operatorname{ker}\left(K_{\theta}^{u}+\delta\right)=$ $\operatorname{ker}\left(K_{\alpha}^{u}+\delta\right)=0$ we conclude that $\operatorname{sign} R_{0}=\operatorname{sign} R_{1}=1$. The result now follows from (3).

\subsection{Orientation of $\mathcal{M}^{*}(X)$}

The mapping torus $X$ gets a canonical Riemannian metric once $\Sigma$ is endowed with a Riemannian metric such that $\tau: \Sigma \rightarrow \Sigma$ is an isometry. Fix a constant lift $u: P \rightarrow P$ and denote by $\bar{P}$ the (trivial) $S U(2)$-bundle over $X$ obtained by first pulling $P$ back to $[0,1] \times \Sigma$ and then identifying the ends via $u$. Given a connection $A$ in $\bar{P}$, consider the respective ASD operator

$$
D_{A}=d_{A}^{*} \oplus d_{A}^{+}: \Omega^{1}(X, \operatorname{ad} \bar{P}) \rightarrow\left(\Omega^{0} \oplus \Omega_{+}^{2}\right)(X, \operatorname{ad} \bar{P}) .
$$

Observe for later use that the mapping torus structure on $X$ can be used to identify both spaces $\Omega^{1}(X$, ad $\bar{P})$ and $\left(\Omega^{0} \oplus \Omega_{+}^{2}\right)(X$, ad $\bar{P})$ with the space $\left(\Omega^{0} \oplus \Omega^{1}\right)^{u}(\mathbb{R} \times \Sigma$, ad $P)$ consisting of $\mathfrak{s u}(2)$-valued 0 - and 1 -forms $\omega(t, x)$ on $\Sigma$ depending on the parameter $t$ in a periodic fashion, $\omega(t+1, x)=u^{*} \omega(t, x)$. 
Here, $u$ can be any lift of $\tau: \Sigma \rightarrow \Sigma$ to the bundle $P$. Note that any periodic map $\mathbb{R} \rightarrow\left(\Omega^{0} \oplus \Omega^{1}\right)_{u}(\Sigma$, ad $P)$ gives rise to an element of $\left(\Omega^{0} \oplus \Omega^{1}\right)^{u}(\mathbb{R} \times$ $\Sigma$, ad $P$ ). A form constructed in this way has the property that $\omega(t, x)=$ $u^{*} \omega(t, x)$; not all elements of $\left(\Omega^{0} \oplus \Omega^{1}\right)^{u}(\mathbb{R} \times \Sigma$; ad $P)$ are of this kind. For any lift $u$ such that $u^{*} A=A$, the operator $D_{A}$ can be viewed as

$$
D_{A}:\left(\Omega^{0} \oplus \Omega^{1}\right)^{u}(\mathbb{R} \times \Sigma, \text { ad } P) \rightarrow\left(\Omega^{0} \oplus \Omega^{1}\right)^{u}(\mathbb{R} \times \Sigma, \text { ad } P) .
$$

Let $\lambda_{X}$ be the determinant bundle of $D_{A}$ over $\mathcal{B}(X)$. This is a real line bundle with the property that, over $\mathcal{M}^{*}(X) \subset \mathcal{B}(X)$, the restriction of $\lambda_{X}$ is isomorphic to the orientation bundle of $\mathcal{M}^{*}(X)$. According to [8], the bundle $\lambda_{X}$ is trivial over $\mathcal{B}(X)$. Therefore, a choice of trivialization of $\lambda_{X}$ (given by a homology orientation, that is, an orientation of $H^{1}(X ; \mathbb{R}) \oplus H_{+}^{2}(X ; \mathbb{R})=$ $\left.H^{1}(X ; \mathbb{R})\right)$ fixes an orientation on $\mathcal{M}^{*}(X)$.

Since $\mathcal{M}^{*}(X)$ is non-degenerate, see Proposition 3.3. it consists of finitely many points. The above orientation convention translates into orienting $[A] \in \mathcal{M}^{*}(X)$ by $\varepsilon\left(D_{\theta}, H, D_{A}\right)$, once orientations of $\operatorname{det} D_{A}$ and $\operatorname{det} D_{\theta}$ are fixed. The former has a canonical orientation because $\operatorname{ker} D_{A}=\operatorname{coker} D_{A}=0$ due to the non-degeneracy. The isomorphisms $\operatorname{ker} D_{\theta}=H^{1}(X ; \operatorname{ad} \theta)$ and coker $D_{\theta}=$ $H^{0}(X ; \operatorname{ad} \theta)$ provide a canonical orientation for $\operatorname{det} D_{\theta}$ once we fix the orientation of the base of the fibration $X \rightarrow S^{1}$.

\subsection{Proof of Theorem 1.1}

We still assume that $\mathcal{R}^{\tau}(\Sigma)$ is non-degenerate. In this case, $\lambda^{\tau}(\Sigma)$ is defined as half the signed count of points in $\mathcal{R}^{\tau}(\Sigma)$ (see Section [5.2), and $\lambda_{F O}(X)$ as one fourth the signed count of points in $\mathcal{M}^{*}(X)$ (see Section 4). According to Proposition 3.1 the restriction map $i^{*}: \mathcal{M}^{*}(X) \rightarrow \mathcal{R}^{\tau}(\Sigma)$ is two-to-one, therefore, to prove the theorem, it is sufficient to show that $i^{*}$ is orientation preserving.

Let $\alpha$ be an equivariant flat connection on $\Sigma$ and choose a constant lift $u: P \rightarrow$ $P$ of the diffeomorphism $\tau$ such that $\alpha=u^{*} \alpha$ and $u^{n}= \pm 1$. By averaging, choose a path $\alpha(s), 0 \leq s \leq 1$, connecting $\theta$ to $\alpha$ such that $\alpha(s)=u^{*} \alpha(s)$ for all $s$. It gives rise to a path of connections $A(s)$ on the product $[0,1] \times \Sigma$ given by the formula $A(s)(t, x)=\alpha(s)(x), x \in \Sigma$, compare with Section 3.1. These define a path of connections on the mapping torus, called again $A(s)$, because $A(s)(1, x)=u^{*} A(s)(0, x)$.

The following proposition is the main step in comparing the orientations. 
Proposition 3.6 Let $A \in \mathcal{M}^{*}(X)$ and $\alpha=i^{*} A \in \mathcal{R}^{\tau}(\Sigma)$ then, for the homotopy $H_{s}=D_{A(s)}-D_{\theta}$ and a small generic $\delta>0$,

$$
\varepsilon\left(D_{\theta}-\delta, H, D_{A}-\delta\right)=\varepsilon\left(K_{\theta}^{u}+\delta, h, K_{\alpha}^{u}+\delta\right) .
$$

Proof We begin by identifying $\operatorname{ker}\left(D_{A(s)}-\delta\right)$ with $\operatorname{ker}\left(K_{\alpha(s)}^{u}+\delta\right)$. Let us fix an $s$, and let $\left\{\psi_{k}\right\}$ be a basis of eigenforms for the operator $K_{\alpha(s)}$,

$$
K_{\alpha(s)} \psi_{k}=\lambda_{k} \psi_{k} .
$$

Any differential form $\omega$ on $X$ can then be written in this basis as

$$
\omega(t, x)=\sum_{k} a_{k}(t) \psi_{k}(x) .
$$

The standard calculation shows that $D_{A(s)}=\partial / \partial t-K_{\alpha(s)}$ hence the equation $\left(D_{A(s)}-\delta\right) \omega=0$ is equivalent to

$$
\sum_{k}\left(a_{k}^{\prime}-\left(\lambda_{k}+\delta\right) a_{k}\right) \psi_{k}=0
$$

or $a_{k}^{\prime}=\left(\lambda_{k}+\delta\right) a_{k}$ for every $k$. Therefore, $a_{k}(t)=a_{k}(0) e^{\left(\lambda_{k}+\delta\right) t}$ and

$$
\omega(t, x)=\sum_{k} a_{k}(0) e^{\left(\lambda_{k}+\delta\right) t} \psi_{k}(x) .
$$

Since $\alpha(s)$ is irreducible, the lift $u$ such that $u^{*} \alpha(s)=\alpha(s)$ is determined uniquely up to a sign, so that the form $\omega$ satisfies the periodic boundary condition $\omega(t+1, x)=u^{*} \omega(t, x)$. On every eigenspace of $K_{\alpha(s)}$ with a fixed eigenvalue $\lambda$, this translates into

$$
u^{*} \sum_{\lambda_{k}=\lambda} a_{k}(0) \psi_{k}(x)=e^{\lambda+\delta} \cdot \sum_{\lambda_{k}=\lambda} a_{k}(0) \psi_{k}(x) .
$$

This means that $e^{\lambda+\delta}$ is an eigenvalue of $u^{*}$. Since $u^{*}$ has finite order and $\lambda+\delta$ is a real number, we must have $\lambda+\delta=0$. Therefore, $\omega$ belongs to the kernel of $K_{\alpha(s)}^{u}+\delta$. Conversely, it is easy to see that $\operatorname{ker}\left(D_{A(s)}-\delta\right)$ is contained in $\operatorname{ker}\left(K_{\alpha(s)}^{u}+\delta\right)$.

A similar argument using the formula $D_{A(s)}^{*}=-\partial / \partial t-K_{\alpha(s)}$ shows that $\operatorname{coker}\left(D_{A(s)}-\delta\right)=\operatorname{ker}\left(K_{\alpha(s)}^{u}+\delta\right)$.

For a small generic $\delta>0$, the spectral curves of $K_{\alpha(s)}^{u}+\delta$ intersect the $s$-axis transversally. Since $H_{s}=D_{A(s)}-D_{\theta}=K_{\theta}^{u}-K_{\alpha(s)}^{u}=-h_{s}$, this implies that the homotopy $H_{s}$ is regular. The proof is now complete. 
Remark 3.7 A similar argument can be used to show that $H_{\tau}^{1}(\Sigma, \operatorname{ad} \alpha)=$ $H^{1}(X ; \operatorname{ad} A)$ thus providing an independent proof of Proposition 3.3 .

To finish the orientation comparison, we need to evaluate $\varepsilon\left(D_{\theta}, H, D_{A}\right)$. To this end, we choose a path of operators from $D_{\theta}$ to $D_{A}$ which consists of three segments. The first segment is $D_{\theta}-s \cdot \delta, 0 \leq s \leq 1$, connecting $D_{\theta}$ to $D_{\theta}-\delta$, the second is $D_{A(s)}-\delta$ as in Proposition [3.6] and the last is $D_{A}-(1-s) \cdot \delta, 0 \leq s \leq 1$, connecting $D_{A}-\delta$ to $D_{A}$. Since the orientation transport is additive, the orientation transports $\varepsilon\left(D_{\theta}, H, D_{A}\right)$ and $\varepsilon\left(D_{\theta}-\delta, H, D_{A}-\delta\right)$, for a small generic $\delta>0$, differ by the product of orientation transports along the first and the last segments. If $\delta$ is small enough, the operators in the last segment have zero kernels, making for the trivial orientation transport. The family $D_{\theta}-s \cdot \delta$ only has kernel at $s=0$. That kernel is isomorphic to $H^{0}(X ; \operatorname{ad} \theta)=\mathfrak{s u}(2)$, with the resonance operator $R_{0}=-\delta \cdot \operatorname{Id}: \mathfrak{s u}(2) \rightarrow \mathfrak{s u}(2)$ so that $\operatorname{det} R_{0}=-\delta^{3}<0$. Finally, note that in formula (3) for the orientation transport, the product of the terms $(-1)^{d_{s}}$ starts at $s=0$. Since dimker $D_{\theta}=3$, we conclude that the orientation transport along the first segment is also equal to +1 , and $i^{*}$ is orientation preserving.

\subsection{A note on the Lefschetz number}

Under certain non-degeneracy assumptions on the character variety $\mathcal{R}^{*}(\Sigma)$, one can express the Furuta-Ohta invariant of a mapping torus in terms of the action of $\tau$ on the Floer homology of $\Sigma$. The standing assumption we make in this subsection is as follows (compare with Section [5):

(*) There is an equivariant admissible perturbation $h$ such that the perturbed moduli space $\mathcal{R}_{h}^{*}(\Sigma)$ is non-degenerate.

Note that this condition is not as strong as requiring that $\mathcal{R}^{*}(\Sigma)$ be nondegenerate, yet it is stronger than is guaranteed by the construction in Section 5 . We will see in Section 9 that condition $(*)$ holds in some interesting examples.

Under the above assumption, there is an action of $\tau^{*}$ on $\mathcal{R}_{h}^{*}(\Sigma)$. For an automorphism $f: V \rightarrow V$ of a graded module $V$, denote by $L(f, V)$ its Lefschetz number. Note that this only requires a $\mathbb{Z}_{2}$-grading, and so makes sense for the action of $\tau$ on the $\mathbb{Z}_{8}$-graded Floer chain complex $I C_{*}(\Sigma)$ and Floer homology groups $I_{*}(\Sigma)$. 
Theorem 3.8 Let $\tau: \Sigma \rightarrow \Sigma$ be an orientation-preserving diffeomorphism such that the non-degeneracy condition $(*)$ holds. Then the Furuta-Ohta invariant of the mapping torus $X$ of $\tau$ is given by

$$
\lambda_{F O}(X)=\frac{1}{2} L\left(\tau, I_{*}(\Sigma)\right) .
$$

Proof Write $\mathcal{R}_{h}^{*}(\Sigma)=\mathcal{R}^{p} \cup \mathcal{R}^{\tau}$ where $\mathcal{R}^{\tau}$ is the fixed point set of $\tau^{*}$ acting on $\mathcal{R}_{h}^{*}(\Sigma)$, and points in $\mathcal{R}^{p}$ are permuted by $\tau^{*}$. The submodules of the Floer chain complex $I C_{*}(\Sigma)$ generated by these sets will be called $I C_{*}^{p}$ and $I C_{*}^{\tau}$. They need not be sub chain complexes; however, the chain map $\tau_{*}: I C_{*}(\Sigma) \rightarrow$ $I C_{*}(\Sigma)$ induced by $\tau_{*}$ preserves these two submodules, as well as the grading.

It follows that (the first equality is the Hopf trace formula; the second is just linear algebra)

$$
L\left(\tau, I_{*}(\Sigma)\right)=L\left(\tau, I C_{*}(\Sigma)\right)=L\left(\tau, I C_{*}^{p}\right)+L\left(\tau, I C_{*}^{\tau}\right) .
$$

Note that by construction, the diagonal entries for $\left(\tau, I C_{*}^{p}\right)$ are all 0 , and the ones for $\left(\tau, I C_{*}^{\tau}\right)$ are \pm 1 , whose signs we now have to figure out.

Let us follow [9, Section 5.4] and orient every generator $\alpha$ in the Floer chain complex by the respective line bundle $\lambda_{W}(\alpha) \otimes \lambda_{W}^{*}(\theta)$, where $W$ is a smooth compact oriented 4-manifold with boundary $\Sigma$, and $\lambda_{W}(\alpha)$ is the determinant bundle over the moduli space of connections on $W$ with limiting value $\alpha$. Now, to compute the sign of $\tau^{*} \alpha$, we simply attach the mapping cylinder $C$ of $\tau$ to $W$. Then $\tau^{*} \alpha$ is oriented by $\lambda_{W \cup C}\left(\tau^{*} \alpha\right) \otimes \lambda_{W \cup C}(\theta)$. By the excision principle, we have

$$
\lambda_{W \cup C}\left(\tau^{*} \alpha\right)=\lambda_{W}(\alpha) \otimes \lambda_{C}\left(\alpha, \tau^{*} \alpha\right)
$$

and $\lambda_{W \cup C}(\theta)=\lambda_{W}(\theta) \otimes 1$. Thus the sign of the diagonal entry in $\left(\tau, I C_{*}^{\tau}\right)$ corresponding to $\alpha$ is $\lambda_{C}\left(\alpha, \tau^{*} \alpha\right)$, which, by excision principle, also orients the respective point in $\mathcal{M}^{*}(X)$ (compare with Section [3.5).

Taking into account the two-to-one correspondence between $\mathcal{M}^{*}(X)$ and $\mathcal{R}^{\tau}(\Sigma)$ (given by Proposition 3.1 and, in the perturbed case, by Proposition [5.31), we obtain the result.

Remark 3.9 The observation that a gauge-theoretic invariant of a mapping torus may be interpreted as a Lefschetz number occurs in several other papers [12, 2, 25]. These works are concerned with mapping tori of surface diffeomorphisms, rather than 4-dimensional mapping tori. Because the moduli spaces of unitary connections (resp. solutions to the Seiberg-Witten equations) 
on a surface are well-understood, the non-degeneracy assumptions in [12, 2] (resp. [25]) are milder than our condition (*).

We conjecture that the equality of $\lambda_{F O}$ and the Lefschetz number holds without any additional smoothness assumptions. It would be of some interest, with regard to the conjecture of Furuta and Ohta, to see some direct relationship between the Lefschetz number and the Rohlin invariant.

\section{Definition of the Furuta-Ohta invariant}

Before we go on to prove Theorem 1.1 in general, we give a detailed description of the class of perturbations needed to properly define the Furuta-Ohta invariant, as such a description is not detailed in [13. The proof of Theorem 1.1 in the next section will then amount to finding sufficiently many equivariant perturbations in this class.

\subsection{Admissible perturbations}

Let $X$ be a $\mathbb{Z}[\mathbb{Z}]$-homology $S^{1} \times S^{3}$. Consider an embedding $\psi: S^{1} \rightarrow X$ and extend it to an embedding $\psi: S^{1} \times N^{3} \rightarrow X$ where $N^{3}$ is an oriented 3 -manifold. Thus for each point $x \in N^{3}$ we have a parallel copy $\psi\left(S^{1} \times\{x\}\right)$ of $\psi: S^{1} \rightarrow X$.

Let $\bar{P}$ be a trivial $S U(2)$-bundle over $X$, and let $\mathcal{A}(X)$ be the affine space of connections in $\bar{P}$. Given $A \in \mathcal{A}(X)$, denote by $\operatorname{hol}_{A}\left(\psi\left(S^{1} \times\{x\}\right), s\right) \in S U(2)$ the holonomy of $A$ around the loop $\psi\left(S^{1} \times\{x\}\right)$ starting at the point $\psi(s, x)$. Let $\Pi: S U(2) \rightarrow \mathfrak{s u}(2)$ be the projection given by

$$
\Pi(u)=u-\frac{1}{2} \operatorname{tr}(u) \cdot \mathrm{Id} .
$$

It is equivariant with respect to the adjoint action on both $S U(2)$ and $\mathfrak{s u}(2)$, therefore, assigning $\Pi \operatorname{hol}_{A}\left(\psi\left(S^{1} \times\{x\}\right), s\right)$ to $\psi(s, x) \in X$ defines a section of ad $\bar{P}=\bar{P} \times$ ad $\mathfrak{s u}(2)$ over $\psi\left(S^{1} \times N^{3}\right)$. Let $\nu \in \Omega_{+}^{2}(X)$ be a self-dual 2-form supported in $\psi\left(S^{1} \times N^{3}\right)$ and define a section

$$
\sigma(\nu, \psi, A) \in \Omega_{+}^{2}(X, \text { ad } \bar{P})
$$

by taking tensor product of $\Pi \operatorname{hol}_{A}\left(\psi\left(S^{1} \times\{x\}\right), s\right)$ with $\nu$ over $\psi\left(S^{1} \times N^{3}\right)$ and letting it be zero otherwise. For fixed $\nu$ and $\psi$, this defines a map $\sigma: \mathcal{A}(X) \rightarrow$ $\Omega_{+}^{2}(X, \operatorname{ad} \bar{P})$ which is equivariant with respect to the gauge group $\mathcal{G}(X)$. 
More generally, let $\Psi=\left\{\psi_{k}\right\}$ be a collection of embedded loops $\psi_{k}: S^{1} \rightarrow X$, $k=1, \ldots, n$, with disjoint images. We will refer to $\Psi$ as a link. Extend $\Psi$ to a collection of embeddings $\psi_{k}: S^{1} \times N_{k}^{3} \rightarrow X$ as above so that the $\psi_{k}\left(S^{1} \times N_{k}^{3}\right)$ are disjoint. For any choice of $n$ smooth functions $\bar{f}_{1}, \ldots, \bar{f}_{n}:[-2,2] \rightarrow \mathbb{R}$ with vanishing derivatives at \pm 2 , define $\sigma: \mathcal{A}(X) \rightarrow \Omega_{+}^{2}(X$, ad $\bar{P})$ by the formula

$$
\sigma(A)=\sum_{k=1}^{n} \partial \bar{f}_{k} \cdot \sigma\left(\nu_{k}, \psi_{k}, A\right),
$$

where $\partial \bar{f}_{k}$ is the function $\bar{f}_{k}^{\prime}$ evaluated at $\operatorname{tr} \operatorname{hol}_{A}\left(\psi_{k}\left(S^{1} \times\{x\}\right), s\right)$, and $\nu_{k}$ are real valued self-dual forms on $X$, each supported in its respective $\psi_{k}\left(S^{1} \times N_{k}^{3}\right)$. We call $\sigma$ an admissible perturbation relative to $\Psi$.

For a fixed choice of the forms $\nu_{1}, \ldots, \nu_{n}$, denote by $\mathcal{F}_{\Psi}$ the space of admissible perturbations relative to $\Psi$ with the $C^{3}$-topology given by the correspondence $\sigma \mapsto\left(\bar{f}_{1}, \ldots, \bar{f}_{n}\right)$.

\subsection{Perturbed ASD connections}

Let $\sigma: \mathcal{A}(X) \rightarrow \Omega_{+}^{2}(X$, ad $\bar{P})$ be an admissible perturbation and define $\zeta_{\sigma}: \mathcal{A}(X) \rightarrow$ $\Omega_{+}^{2}(X$, ad $\bar{P})$ by the formula

$$
\zeta_{\sigma}(A)=F_{+}(A)+\sigma(A) .
$$

A connection $A$ is called perturbed $A S D$ if $\zeta_{\sigma}(A)=0$. The moduli space of perturbed ASD connections will be denoted by $\mathcal{M}_{\sigma}(X)$ so that $\mathcal{M}_{\sigma}(X)=$ $\zeta_{\sigma}^{-1}(0) / \mathcal{G}(X)$.

If $\sigma=0$ then $\mathcal{M}_{\sigma}(X)$ coincides with the flat moduli space $\mathcal{M}(X)$. Since $\pi_{1}(X)$ is finitely presented, $\mathcal{M}(X)$ is a compact real algebraic variety. According to [13, reducible flat connections form a connected component in $\mathcal{M}(X)$, therefore the moduli space $\mathcal{M}^{*}(X)$ of irreducible flat connections is also compact. We are only interested in irreducible connections hence we will not perturb the reducible part of $\mathcal{M}(X)$. Note that small enough perturbations do not create new reducible connections. Denote by $\mathcal{M}_{\sigma}^{*}(X)$ the moduli space of irreducible perturbed ASD connections; for a sufficiently small $\sigma$, it is compact.

The local structure of $\mathcal{M}_{\sigma}^{*}(X)$ near a point $[A] \in \mathcal{M}_{\sigma}^{*}(X)$ is described by the deformation complex

$$
\Omega^{0}(X, \text { ad } \bar{P}) \stackrel{d_{A}}{\longrightarrow} \Omega^{1}(X, \text { ad } \bar{P}) \stackrel{d_{A}^{+}+D \sigma(A)}{\longrightarrow} \Omega_{+}^{2}(X, \text { ad } \bar{P}) .
$$

We call $\mathcal{M}_{\sigma}^{*}(X)$ non-degenerate at $[A] \in \mathcal{M}_{\sigma}^{*}(X)$ if the second cohomology of the above complex vanishes, that is, $H_{\sigma,+}^{2}(X ; \operatorname{ad} A)=\operatorname{coker}\left(d_{A}^{+}+D \sigma(A)\right)=0$. 
Since $A$ is irreducible, an equivalent condition is vanishing of $\operatorname{coker}\left(D_{A}^{\sigma}\right)$, where $D_{A}^{\sigma}=D_{A}+D \sigma(A)$ is the perturbed ASD operator ([6). We call $\mathcal{M}_{\sigma}^{*}(X)$ non-degenerate if it is non-degenerate at all $[A] \in \mathcal{M}_{\sigma}^{*}(X)$. If $\mathcal{M}_{\sigma}^{*}(X)$ is non-degenerate, it consists of finitely many points oriented by the orientation transport $\varepsilon\left(D_{\theta}^{\sigma}, H, D_{A}^{\sigma}\right)=\varepsilon\left(D_{\theta}, H, D_{A}^{\sigma}\right)$, compare with Section 3.5 .

\subsection{Abundance of perturbations}

We wish to show that we have enough admissible perturbations $\sigma$ to find a non-degenerate $\mathcal{M}_{\sigma}^{*}(X)$. A link $\Psi=\left\{\psi_{k}\right\}$ will be called abundant at an ASD connection $A$ if there exist self dual 2-forms $\nu_{k}$ supported in $\psi_{k}\left(S^{1} \times N_{k}^{3}\right)$ such that the sections $\sigma\left(\nu_{k}, \psi_{k}, A\right)$ span the vector space $H_{+}^{2}(X ; \operatorname{ad} A)$. Note that if a link $\Psi$ is abundant at $A$ it is also abundant at any ASD connection gauge equivalent to $A$.

Lemma 4.1 There exists a link $\Psi$ which is abundant at all $[A] \in \mathcal{M}^{*}(X)$.

Proof That there is a link which is abundant at any given $[A] \in \mathcal{M}^{*}(X)$ is immediate from Lemma (2.5) of [8]. Abundance is an open condition with respect to $A$ hence existence of the universal link $\Psi$ follows from compactness of $\mathcal{M}^{*}(X)$.

Proposition 4.2 For a small generic admissible perturbation $\sigma$, the moduli space $\mathcal{M}_{\sigma}^{*}(X)$ is non-degenerate.

Proof Let us fix an abundant link $\Psi$ and consider the map $\Phi: \mathcal{B}^{*}(X) \times \mathcal{F}_{\Psi} \rightarrow$ $\Omega_{+}^{2}(X$, ad $\bar{P})$ given by the formula

$$
\Phi(A, \bar{f})=F_{+}(A)+\sum_{k=1}^{n} \partial \bar{f}_{k} \cdot \sigma\left(\nu_{k}, \psi_{k}, A\right)
$$

where $\bar{f}=\left(\bar{f}_{1}, \ldots, \bar{f}_{n}\right) \in \mathcal{F}_{\Psi}$. The partial derivative of this map with respect to $A$ has cokernel $H_{+}^{2}(X ; \operatorname{ad} A)$, while the partial derivative with respect to $\bar{f}$ is onto $H_{+}^{2}(X ; \operatorname{ad} A)$ according to Lemma 4.1

Therefore, $\Phi$ is a submersion; in particular, $\Phi^{-1}(0)$ is locally a smooth manifold. Consider projection of $\Phi^{-1}(0) \subset \mathcal{B}^{*}(X) \times \mathcal{F}_{\Psi}$ onto the second factor. By the Sard-Smale theorem, this projection is a submersion for a dense set of $\bar{f}$ within a sufficiently small neighborhood of zero in $\mathcal{F}_{\Psi}$. Since the pre-image of $\bar{f}$ is the moduli space $\mathcal{M}_{\sigma}^{*}(X)$ with $\sigma(A)=\sum \partial \bar{f}_{k} \cdot \sigma\left(\nu_{k}, \psi_{k}, A\right)$, we are finished. 


\subsection{Definition of the invariant}

The Furuta-Ohta invariant is defined as one fourth of the signed count of points in a non-degenerate moduli space $\mathcal{M}_{\sigma}^{*}(X)$. That it is well defined follows from the standard cobordism argument: for any generic path of small admissible perturbations $\sigma(t), t \in[0,1]$, the parameterized moduli space

$$
\overline{\mathcal{M}}^{*}(X)=\bigcup_{t \in[0,1]} \mathcal{M}_{\sigma(t)}^{*}(X) \times\{t\}
$$

is a compact oriented cobordism between $\mathcal{M}_{\sigma(0)}^{*}(X)$ and $\mathcal{M}_{\sigma(1)}^{*}(X)$. The cobor$\operatorname{dism} \overline{\mathcal{M}}^{*}(X)$ is compact because the condition $H_{*}(\tilde{X} ; \mathbb{Z})=H_{*}\left(S^{3} ; \mathbb{Z}\right)$ ensures that the reducible connections are isolated in the flat moduli space, see [13].

\section{The perturbed case}

In this section, we prove Theorem 1.1 without assuming that $\mathcal{R}^{\tau}(\Sigma)$ is nondegenerate. We begin by recalling the definition of the equivariant Casson invariant, which was given in [7] for finite order diffeomorphisms $\tau$ having fixed points, and then extending it to cover the fixed point free $\tau$ (still of finite order). Theorem 1.1 follows after matching perturbations in the two theories.

\subsection{Perturbing $\mathcal{R}^{\tau}(\Sigma)$}

The decomposition (2) splits $\mathcal{R}^{\tau}(\Sigma)$ into a union of $\mathcal{R}^{u}(\Sigma)=\mathcal{R}^{\tau}(\Sigma) \cap \mathcal{B}^{u}(\Sigma)$ each of which consists of (the gauge equivalence classes of) flat connections $\alpha$ such that $u^{*} \alpha=\alpha$, for a constant lift $u: P \rightarrow P$. Clearly, it is sufficient to do perturbations on each of the $\mathcal{B}^{u}(\Sigma)$ separately, hence we will fix a constant lift $u$ from the beginning.

Let $\left\{\gamma_{j}\right\}$ be a link in $\Sigma$, that is, a collection $\gamma_{j}: S^{1} \times D^{2} \rightarrow \Sigma, j=1, \ldots, n$, of embeddings with disjoint images. For any $\alpha \in \mathcal{A}(\Sigma)$, denote by $\operatorname{hol}_{\alpha}\left(\gamma_{j}\left(S^{1} \times\right.\right.$ $\{z\}), s) \in S U(2)$ the holonomy of $\alpha$ around the loop $\gamma_{j}\left(S^{1} \times\{z\}\right)$ starting at the point $\gamma_{j}(s, z)$. Let $\eta(z)$ be a smooth rotationally symmetric bump function on the disc $D^{2}$ with support away from the boundary of $D^{2}$ and with integral one, and let $f_{j}: S U(2) \rightarrow \mathbb{R}$ be a collection of smooth functions invariant with respect to conjugation. Following [30, define an admissible perturbation $h: \mathcal{A}^{u}(\Sigma) \rightarrow \mathbb{R}$ by the formula

$$
h(\alpha)=\sum_{j=1}^{n} \int_{D^{2}} f_{j}\left(\operatorname{hol}_{\alpha}\left(\gamma_{j}\left(S^{1} \times\{z\}\right), s\right)\right) \eta(z) d^{2} z .
$$


The conjugation invariance of the $f_{j}$ ensures that the function $h$ does not depend on $s$. The action of the gauge group only changes holonomies around $\gamma_{j}\left(S^{1} \times\{z\}\right)$ within their $S U(2)$ conjugacy classes. Therefore, the admissible perturbation $h$ is well defined on $\mathcal{B}^{u}(\Sigma)$.

Suppose that the link $\left\{\gamma_{j}\right\}$ is invariant with respect to the action of $\tau$ and that $f_{j}=f_{m}$ whenever $\tau_{*} \gamma_{j}=\gamma_{m}$. Then formula (9) defines an admissible perturbation $h$ such that $h\left(\tilde{\tau}^{*} \alpha\right)=h(\alpha)$ for any lift $\tilde{\tau}$. We will call $h$ an equivariant admissible perturbation. The formula

$$
\zeta_{h}^{u}(\alpha)=* F(\alpha)+\nabla h(\alpha)
$$

where $\nabla h$ is the $L^{2}$-gradient of $h$, defines a map $\zeta_{h}^{u}: \mathcal{A}^{u}(\Sigma) \rightarrow \Omega_{u}^{1}(\Sigma, \operatorname{ad} P)$ where $\omega \in \Omega_{u}^{1}(\Sigma$, ad $P)$ if and only if $u^{*} \omega=\omega$, see Section 3.4.

A connection $\alpha \in \mathcal{A}^{u}(\Sigma)$ is called perturbed flat if $\zeta_{h}^{u}(\alpha)=0$. The moduli space of the perturbed equivariant flat connections will be denoted by $\mathcal{R}_{h}^{u}(\Sigma)$ so that $\mathcal{R}_{h}^{u}(\Sigma)=\left(\zeta_{h}^{u}\right)^{-1}(0) / \mathcal{G}^{u}(\Sigma)$. If $h=0$ then $\mathcal{R}_{h}^{u}(\Sigma)$ coincides with the moduli space $\mathcal{R}^{u}(\Sigma)$ of equivariant flat connections.

The moduli space $\mathcal{R}_{h}^{u}(\Sigma)$ is called non-degenerate if $H_{h, \tau}^{1}(\Sigma ; \operatorname{ad} \alpha)=\operatorname{ker}\left(d_{\alpha} \zeta_{h}^{u}\right)$ $\cap \operatorname{ker} d_{\alpha}^{*}$ vanishes for all $[\alpha] \in \mathcal{R}_{h}^{u}(\Sigma)$. After a minor modification to cover the case of empty $\operatorname{Fix}(\tau)$, the argument of [7], Section 3.8, shows that there exist small equivariant admissible perturbations $h: \mathcal{A}(\Sigma) \rightarrow \mathbb{R}$ making all the moduli spaces $\mathcal{R}_{h}^{u}(\Sigma)$ non-degenerate. For such a perturbation, the space $\mathcal{R}_{h}^{\tau}(\Sigma)$, which is a union of the $\mathcal{R}_{h}^{u}(\Sigma)$, is smooth compact manifold of dimension zero.

\subsection{Definition of the equivariant Casson invariant}

Let $\mathcal{R}^{\tau}(\Sigma)$ be the equivariant flat moduli space, and choose (if necessary) a small equivariant admissible perturbation $h$ such that $\mathcal{R}_{h}^{\tau}(\Sigma)$ is non-degenerate. Define the equivariant Casson invariant by the formula

$$
\lambda^{\tau}(\Sigma)=\frac{1}{2} \sum_{\alpha \in \mathcal{R}_{h}^{\tau}(\Sigma)}(-1)^{\mu^{\tau}(\alpha)},
$$

where $\mu^{\tau}(\alpha)$ is the equivariant spectral flow of the perturbed odd signature operators $K_{h, \alpha(s)}=K_{\alpha(s)}+\operatorname{Hess}_{\alpha(s)} h$ as in Section 3.4 If $\tau$ has fixed points, this invariant coincides with the equivariant Casson invariant defined in [7].

Proposition 5.1 The invariant $\lambda^{\tau}(\Sigma)$ is well defined as an integer valued invariant of the pair $(\Sigma, \tau)$. 
Proof This was proved in [7] in the case when $\tau$ has fixed points. For a fixed point free $\tau$, the result is immediate from Theorem [7.4 which expresses $\lambda^{\tau}(\Sigma)$ in terms of the Boyer-Nicas type invariants of $\Sigma / \tau$.

\subsection{Matching the perturbations}

Let $X$ be the mapping torus of $\tau: \Sigma \rightarrow \Sigma$. If $\mathcal{M}^{*}(X)$ happens to be degenerate then so is the flat moduli space $\mathcal{R}^{\tau}(\Sigma)$, see Proposition 3.3. Our goal in this section will be to perturb $\mathcal{M}^{*}(X)$ and $\mathcal{R}^{\tau}(\Sigma)$ in a consistent manner so as to keep the two-to-one correspondence between them which existed in the unperturbed case.

Let us fix a constant lift $u: P \rightarrow P$ and let $\bar{P}$ be the trivial $S U(2)-$ bundle over $X$ obtained by pulling $P$ back to $[0,1] \times \Sigma$ and identifying the ends via $u$. The maps $\pi: \mathcal{A}^{u}(\Sigma) \rightarrow \mathcal{A}^{*}(X)$, see Remark 3.2. can be included into the commutative diagram

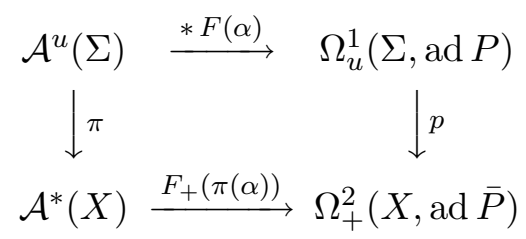

where $p$ is defined as follows. Given $\omega \in \Omega_{u}^{1}(\Sigma$, ad $P)$, pull the 2 -form $* \omega$ back to $[0,1] \times \Sigma$ and identify the ends via $u^{*}$. The form $p(w)$ is then the self-dual part of this 2 -form. The zero set of $* F(\alpha)$ is the flat moduli space $\mathcal{R}^{u}(\Sigma)$, and $\pi$ establishes a one-to-two correspondence between the union of all the $\mathcal{R}^{u}(\Sigma)$ and the moduli space $\mathcal{M}^{*}(X)$, see Proposition 3.1

Proposition 5.2 There exists an equivariant admissible perturbation $h$ and an admissible perturbation $\sigma$ such that, for every $u$, the diagram

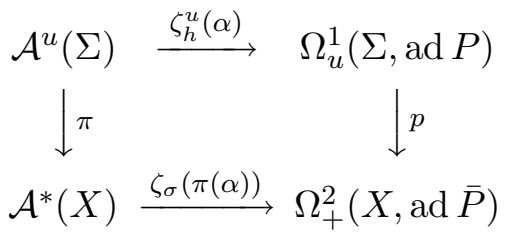

commutes, and both moduli spaces $\mathcal{R}_{h}^{\tau}(\Sigma)$ and $\mathcal{M}_{\sigma}^{*}(X)$ are non-degenerate. 
Proof We start with an equivariant admissible perturbation $h: \mathcal{A}^{u}(\Sigma) \rightarrow \mathbb{R}$ and evaluate explicitly $\zeta_{h}^{u}(\alpha)=* F(\alpha)+\nabla h(\alpha)$. Write each of the $f_{j}: S U(2) \rightarrow$ $\mathbb{R}$ as $f_{j}=\bar{f}_{j} \circ \operatorname{tr}$ for a smooth function $\bar{f}_{j}:[-2,2] \rightarrow \mathbb{R}$. Then, according to [16], the gradient $\nabla h(\alpha)$ is the 1 -form

$$
\nabla h(\alpha)=\sum_{j=1}^{n} \bar{f}_{j}^{\prime}\left(\operatorname{trhol}_{\alpha}\left(\gamma_{j}\left(S^{1} \times\{z\}, s\right)\right)\right) \cdot \Pi \operatorname{hol}_{\alpha}\left(\gamma_{j}\left(S^{1} \times\{z\}\right), s\right) \eta(z) d s
$$

where each of the summands is understood to have support in its respective copy of $\gamma_{j}\left(S^{1} \times D^{2}\right)$. Next, each orbit of the $\mathbb{Z}_{n}$-action induced by $\tau$ on $\left\{\gamma_{j}\right\}$ gives rise to a copy of $S^{1} \times S^{1} \times D^{2}$ and hence to the link $\psi_{k}: S^{1} \times N_{k}^{3} \rightarrow X$ with $S^{1}$-direction corresponding to $\gamma_{j}\left(S^{1} \times\{0\}\right)$ and $N_{k}^{3}=S^{1} \times D^{2}$. For any connection $A$ over $X$ set $\nu_{k}=p(\eta(z) d s)$ and define $\sigma$ as in (8). An easy calculation shows that $\operatorname{hol}_{\pi(\alpha)}\left(\psi_{k}\left(S^{1} \times\{(t, z)\}\right), s\right)=\operatorname{hol}_{\alpha}\left(\gamma_{j}\left(S^{1} \times\{z\}\right), s\right)$, for a respective $j$ in the $\mathbb{Z}_{n}$-orbit which gives rise to $\psi_{k}$. Therefore, $\sigma$ makes the diagram (10) commute.

The second statement follows from the fact that the map $p^{*}: H_{h, \tau}^{1}(\Sigma ;$ ad $\alpha) \rightarrow$ $H_{\sigma,+}^{2}(X ; \operatorname{ad} \pi(\alpha))$ is an isomorphism. This follows via a minor modification of the proof of Proposition 3.6. the crucial observation being that $D_{A}^{\sigma}=\partial / \partial t-$ $K_{h, \alpha}$, compare with Remark 3.7.

Let us fix $h$ and $\sigma$ as in Proposition 5.2. The following result is a perturbed analogue of Proposition 3.1

Proposition 5.3 The map $\pi$ provides a one-to-two correspondence between $\mathcal{R}_{h}^{\tau}(\Sigma)$ and $\mathcal{M}_{\sigma}^{*}(X)$.

Proof We only need to check that all connections in $\mathcal{M}_{\sigma}^{*}(X)$ are of the form $\pi(\alpha)$, up to gauge equivalence. Given a connection $A$ over $X$, put it into temporal gauge over $[0,1] \times \Sigma$ so that $A=\{A(t)\}$. The connections $A(0)$ and $A(1)$ will be gauge equivalent (via the holonomy along $[0,1] \times\{x\}$ ) although not necessarily equal. Let $\delta(\alpha)$ be the pull-back of $* \nabla h(\alpha)$ over each of the product regions $[0,1] \times \gamma_{j}\left(S^{1} \times D^{2}\right)$, and zero otherwise. Then $\sigma(A)=\delta(A)_{+}$ so that the connection $A$ is perturbed ASD if and only if $F_{+}(A)+\delta_{+}(A)=0$.

We wish to show first that $A$ is perturbed flat, that is, $F(A)+\delta(A)=0$. To this end, calculate 


$$
\begin{aligned}
\|F(A)+\delta(A)\|^{2} & =-\int_{X} \operatorname{tr}(F(A)+\delta(A)) \wedge *(F(A)+\delta(A)) \\
& =\int_{X} \operatorname{tr}(F(A)+\delta(A)) \wedge(F(A)+\delta(A)) \\
& =\int_{X} \operatorname{tr}(F(A) \wedge F(A))+2 \int_{X} \operatorname{tr}(F(A) \wedge \delta(A))
\end{aligned}
$$

since $\delta(A) \wedge \delta(A)=0$. The first integral vanishes by the Chern-Weil theory. To compute the second, pull back to $[0,1] \times \Sigma$ where we write $F(A)=d t \wedge$ $A^{\prime}(t)+F(A(t))$ so that $F(A) \wedge \delta(A)=d t \wedge A^{\prime}(t) \wedge \delta(A)$. First we integrate over $\Sigma$ to obtain

$$
\int_{\Sigma} \operatorname{tr}(F(A) \wedge \delta(A))=\int_{\Sigma} \operatorname{tr}\left(A^{\prime}(t) \wedge * \nabla h(A(t))\right)=\frac{d}{d t} h(A(t)) d t .
$$

Integration with respect to $t$ now yields

$$
\int_{X} \operatorname{tr}(F(A) \wedge \delta(A))=\int_{0}^{1} \frac{d}{d t} h(A(t)) d t=h(A(1))-h(A(0))=0
$$

since $h$ is invariant with respect to gauge transformations.

Now, since $A$ is perturbed flat, it satisfies the equation $d t \wedge A^{\prime}(t)+F(A(t))+$ $* \nabla h(A(t))=0$ over $[0,1] \times \Sigma$ with periodic boundary conditions. Therefore, $A(t)=\alpha$ for all $t$, where $\alpha \in \mathcal{A}^{u}(\Sigma)$ is a perturbed flat connection, so that $A=\pi(\alpha)$ after a gauge transformation if necessary.

\subsection{Proof of Theorem 1.1}

Choose perturbations $h$ and $\sigma$ as in Proposition [5.2, then $\mathcal{M}_{\sigma}^{*}(X)$ and $\mathcal{R}_{h}^{\tau}(\Sigma)$ are in two-to-one correspondence and are both non-degenerate. Moreover, in

temporal gauge, $D_{A}^{\sigma}=\partial / \partial t-K_{h, A(t)}$ where $K_{h, A(t)}$ is the perturbed operator $K_{A(t)}$. The same argument as in the unperturbed case now shows that that the corresponding points in $\mathcal{M}_{\sigma}^{*}(X)$ and $\mathcal{R}_{h}^{\tau}(\Sigma)$ are counted with the same sign. This completes the proof of Theorem [1.1]

\section{The equivariant Casson invariant: $\operatorname{Fix}(\tau) \neq \emptyset$}

In the case of non-empty $\operatorname{Fix}(\tau)$, the equivariant Casson invariant can be easily evaluated using an explicit formula proved in [7]. In this case, the quotient 
space $\Sigma^{\prime}=\Sigma / \tau$ is an integral homology sphere, and the projection $\Sigma \rightarrow \Sigma^{\prime}$ is an $n$-fold branched cover with branch set a knot $k \subset \Sigma^{\prime}$. According to Theorem 1 of [7],

$$
\lambda^{\tau}(\Sigma)=n \cdot \lambda\left(\Sigma^{\prime}\right)+\frac{1}{8} \sum_{m=0}^{n-1} \operatorname{sign}^{m / n} k,
$$

where $\operatorname{sign}^{\alpha} k$ is the Tristram-Levine equivariant knot signature, defined as the signature of the Hermitian form $(1-\exp (2 \pi i \alpha)) S+(1-\exp (-2 \pi i \alpha)) S^{t}$ for any choice of a Seifert matrix $S$ of $k$.

\section{$7 \quad$ The equivariant Casson invariant: $\operatorname{Fix}(\tau)=\emptyset$}

In this section, we obtain a formula for $\lambda^{\tau}(\Sigma)$ in the case of a fixed point free $\tau$. We first express $\lambda^{\tau}(\Sigma)$ in terms of certain Boyer-Nicas type invariants (which extend invariants of [3] and [4]), and then identify the latter via topology. We will be using interchangeably $G$-gauge theories with $G=S O(3), S U(2)$, and $U(2)$; to keep better track of $G$ we will include it in our notations - for example, $\mathcal{R}(\Sigma, S U(2))$ will mean the moduli space of flat $S U(2)$-connections etc.

\subsection{Identifying moduli spaces}

Let $P$ be a trivialized $S U(2)$-bundle over $\Sigma$. According to Section 2.3, the equivalence classes of lifts $\tilde{\tau}: P \rightarrow P$ are classified by the group $H^{2}\left(\Sigma^{\prime} ; \mathbb{Z}_{2}\right)$. The equivalence class of lifts corresponding to $w \in H^{2}\left(\Sigma^{\prime} ; \mathbb{Z}_{2}\right)$ will be denoted by $\tau_{w}$. The decomposition (11) then takes the form

$$
\mathcal{B}^{\tau}(\Sigma, S U(2))=\bigsqcup_{w} \mathcal{B}_{w}^{\tau}(\Sigma, S U(2))
$$

where $\mathcal{B}_{w}^{\tau}(\Sigma, S U(2))$ consists of irreducible $S U(2)$-connections $A$ in $P$ such that $\tau_{w}^{*} A=A$, modulo the group of gauge transformations $g: P \rightarrow P$ such that $g \tau_{w}= \pm \tau_{w} g$. Since $H^{2}\left(\Sigma^{\prime} ; \mathbb{Z}_{2}\right)$ is trivial if $n$ is odd and is equal to $\mathbb{Z}_{2}$ if $n$ is even, we have at most two different equivalence classes of lifts, $\tau_{0}$ and $\tau_{1}$. We will fix constant lifts within each class so that $\tau_{0}(x, y)=(\tau(x), y)$ and $\tau_{1}(x, y)=(\tau(x), u \cdot y)$ where $u \in S U(2)$ is such that $u^{n}=-1$, see Section 2.3 .

For any choice of $\tau_{w}$, the quotient bundle $P_{w}^{\prime}=P / \tau_{w}$ over $\Sigma^{\prime}=\Sigma / \tau$ is an $S O(3)$-bundle with $w_{2}\left(P_{w}^{\prime}\right)=w$. It has a natural smooth structure such that the quotient map $\pi: P \rightarrow P_{w}^{\prime}$ is a smooth bundle morphism. Denote 
by $\mathcal{B}_{w}^{*}\left(\Sigma^{\prime}, S O(3)\right)$ the space of the gauge equivalence classes of irreducible connections in $P_{w}^{\prime}$. Let $\mathcal{R}_{w}^{*}\left(\Sigma^{\prime}, S O(3)\right) \subset \mathcal{B}_{w}^{*}\left(\Sigma^{\prime}, S O(3)\right)$ and $\mathcal{R}_{w}^{\tau}(\Sigma, S U(2)) \subset$ $\mathcal{B}_{w}^{\tau}(\Sigma, S U(2))$ be the respective moduli spaces of irreducible flat connections.

Proposition 7.1 The pull back of connections via $\pi$ induces a homeomorphism between $\mathcal{R}_{w}^{*}\left(\Sigma^{\prime}, S O(3)\right)$ and $\mathcal{R}_{w}^{\tau}(\Sigma, S U(2))$.

Proof First note that the $S U(2)$ and $S O(3)$ gauge theories on $\Sigma$ are equivalent because $\Sigma$ is an integral homology sphere. The result then essentially follows from the discussion in Section 2.3, after we check that the pull back $\pi^{*} \alpha^{\prime}$ of an irreducible flat connection $\alpha^{\prime}$ in $P_{w}^{\prime}$ is irreducible. Let $\alpha^{\prime}: \pi_{1}\left(\Sigma^{\prime}\right) \rightarrow S O(3)$ be the holonomy representation of $\alpha^{\prime}$. Its pull back via $\pi_{1}(\Sigma) \rightarrow \pi_{1}\left(\Sigma^{\prime}\right)$ is the holonomy representation of $\pi^{*} \alpha^{\prime}$. Suppose that $\pi^{*} \alpha^{\prime}$ is reducible, then $\pi^{*} \alpha^{\prime}$ is trivial because $\Sigma$ is an integral homology sphere. This means that $\alpha^{\prime}$ factors through $\pi_{1}\left(\Sigma^{\prime}\right) / \pi_{1}(\Sigma)=\mathbb{Z}_{n}$ and hence is also reducible, a contradiction.

Remark 7.2 If $n$ is odd, we have just one $S O(3)$-bundle on $\Sigma^{\prime}$ which is trivial and hence admits a unique $S U(2)$-lift. In this case, Proposition 7.1 also establishes a bijective correspondence at the level of flat $S U(2)$-connections.

\subsection{The Boyer-Nicas type invariants}

Let $\Sigma^{\prime}=\Sigma / \tau$ as before. Evidently, $\Sigma^{\prime}$ is cyclically finite in the terminology of [3] ie, every cyclic cover of $\Sigma^{\prime}$ is a rational homology sphere. In this situation, Boyer and Nicas defined an integer valued invariant, by counting irreducible $S U(2)$-representations, with signs defined using a Heegaard splitting as in Casson's original work. We will give a definition of the signs in terms of spectral flow, as in Taubes' work [30.

The definition, slightly extended to the case of $S O(3)$-bundles, is as follows. Fix a $w \in H^{2}\left(\Sigma^{\prime} ; \mathbb{Z}_{2}\right)$ and consider the moduli space $\mathcal{R}_{w}^{*}\left(\Sigma^{\prime}, S O(3)\right)$ of the (gauge equivalence classes of) irreducible flat connections in an $S O(3)$-bundle $P_{w}^{\prime}$ over $\Sigma^{\prime}$ having $w_{2}\left(P_{w}^{\prime}\right)=w$. We say that $\mathcal{R}_{w}^{*}\left(\Sigma^{\prime}, S O(3)\right)$ is non-degenerate if, for every $\alpha^{\prime} \in \mathcal{R}_{w}^{*}\left(\Sigma^{\prime}, S O(3)\right)$, the cohomology $H^{1}\left(\Sigma^{\prime}\right.$; ad $\left.\alpha^{\prime}\right)$ vanishes. If $\mathcal{R}_{w}^{*}\left(\Sigma^{\prime}, S O(3)\right)$ is non-degenerate, define the Boyer-Nicas type invariant

$$
\lambda_{w}\left(\Sigma^{\prime}\right)=\frac{1}{2} \sum_{\alpha^{\prime} \in \mathcal{R}_{w}^{*}\left(\Sigma^{\prime}, S O(3)\right)}(-1)^{\mu\left(\alpha^{\prime}\right)},
$$

where $\mu\left(\alpha^{\prime}\right)$ is the spectral flow of the odd signature operator (44) along a generic path connecting $\theta_{w}$ to $\alpha^{\prime}$. Here, $\theta_{0}$ is the product connection in the 
trivial bundle $P_{0}^{\prime}$, and $\theta_{1}$ is the (non-trivial) flat reducible connection in $P_{1}^{\prime}$ with holonomy

$$
\pi_{1}\left(\Sigma^{\prime}\right) \longrightarrow H_{1}\left(\Sigma^{\prime} ; \mathbb{Z}_{2}\right)=\mathbb{Z}_{2} \stackrel{\beta}{\longrightarrow} S O(3),
$$

where $\beta$ maps the generator of $\mathbb{Z}_{2}$ to the diagonal matrix $\operatorname{diag}(1,-1,-1) \in$ $S O(3)$.

If $\mathcal{R}_{w}^{*}\left(\Sigma^{\prime}, S O(3)\right)$ fails to be non-degenerate, it needs to be perturbed first. In this case, the invariant is best described in terms of the moduli space of projectively flat $U(2)$-connections : roughly speaking, $S O(3)$ perturbations are $H^{1}\left(\Sigma^{\prime} ; \mathbb{Z}_{2}\right)$-equivariant $U(2)$ perturbations. We give a brief outline of the construction below and refer to 24 for details.

For each $w \in H^{2}\left(\Sigma^{\prime} ; \mathbb{Z}_{2}\right)$ choose a $U(2)$-bundle $\tilde{P}_{w}^{\prime}$ whose associated $S O(3)$ bundle is $P_{w}^{\prime}$ (such a bundle exists because $H^{3}\left(\Sigma^{\prime} ; \mathbb{Z}\right)$ is torsion free). Then $c_{1}\left(\tilde{P}_{w}^{\prime}\right)=w(\bmod 2)$, and we make our choice so that $\tilde{P}_{w}^{\prime}$ is a trivial bundle whenever $w=0(\bmod 2)$. Every connection on $\tilde{P}_{w}^{\prime}$ induces and is induced by unique connections on $P_{w}^{\prime}$ and on the line bundle $\operatorname{det} \tilde{P}_{w}^{\prime}$.

Let us fix a connection $C$ on $\operatorname{det} \tilde{P}_{w}^{\prime}$ and let $\mathcal{A}_{w}\left(\Sigma^{\prime}, U(2)\right)$ be the space of connections on $\tilde{P}_{w}^{\prime}$ compatible with $C$. If $w=0(\bmod 2)$ we choose $C$ to be the trivial connection; note that $C$ plays no geometric role as different choices lead to equivalent theories. Let $\mathcal{B}_{w}\left(\Sigma^{\prime}, U(2)\right)$ be the quotient space of $\mathcal{A}_{w}\left(\Sigma^{\prime}, U(2)\right)$ by the action of gauge group consisting of unitary automorphisms of $\tilde{P}_{w}^{\prime}$ of determinant one. Contained in $\mathcal{B}_{w}\left(\Sigma^{\prime}, U(2)\right)$ is the moduli space $\mathcal{R}_{w}\left(\Sigma^{\prime}, U(2)\right)$ of projectively flat connections in $\tilde{P}_{w}^{\prime}$. There is a natural action of the group $H^{1}\left(\Sigma^{\prime} ; \mathbb{Z}_{2}\right)$ on both of the above spaces, with quotients $\mathcal{B}_{w}\left(\Sigma^{\prime}, S O(3)\right)$ and $\mathcal{R}_{w}\left(\Sigma^{\prime}, S O(3)\right)$, respectively.

Lemma 7.3 The action of $H^{1}\left(\Sigma^{\prime} ; \mathbb{Z}_{2}\right)$ on $\mathcal{R}_{w}^{*}\left(\Sigma^{\prime}, U(2)\right)$ is free, and its orbit space is $\mathcal{R}_{w}^{*}\left(\Sigma^{\prime}, S O(3)\right)$.

Proof If $n$ is odd the group $H^{1}\left(\Sigma^{\prime} ; \mathbb{Z}_{2}\right)$ vanishes therefore we may assume from the beginning that $n$ is even so that $H^{1}\left(\Sigma^{\prime} ; \mathbb{Z}_{2}\right)=\mathbb{Z}_{2}$.

First let $w=0(\bmod 2)$ then $\mathcal{R}_{w}^{*}\left(\Sigma^{\prime}, U(2)\right)=\mathcal{R}^{*}\left(\Sigma^{\prime}, S U(2)\right)$. The action of $\chi \in H^{1}\left(\Sigma^{\prime} ; \mathbb{Z}_{2}\right)$ viewed as a homomorphism $\chi: \pi_{1} \Sigma^{\prime} \rightarrow \mathbb{Z}_{2}=\{ \pm 1\}$ is given by $\alpha^{\prime} \mapsto \chi \cdot \alpha^{\prime}$. If the conjugacy class of $\alpha^{\prime}$ is fixed by $\chi \neq 1$ then $\chi \alpha^{\prime}=u \alpha^{\prime} u^{-1}$ for some $u \in S U(2)$ such that $u^{2}=-1$. This means that $\alpha^{\prime}$ is a binary dihedral representation and hence its lift $\pi^{*} \alpha^{\prime}: \pi_{1} \Sigma \rightarrow S U(2)$ is reducible. The fact that $\Sigma$ is an integral homology sphere implies that $\pi^{*} \alpha^{\prime}$ is trivial, which means that $\alpha^{\prime}$ cannot be irreducible. 
Now suppose that $w=1(\bmod 2)$ then $\mathcal{R}_{w}^{*}\left(\Sigma^{\prime}, U(2)\right)$ consists of irreducible projective representations $\alpha^{\prime}: \pi_{1} \Sigma^{\prime} \rightarrow S U(2)$ with $w_{2}\left(\operatorname{ad} \alpha^{\prime}\right)=1(\bmod 2)$. Arguing as above, we conclude that the fixed points of a non-trivial $\chi \in H^{1}\left(\Sigma^{\prime} ; \mathbb{Z}_{2}\right)$ are the binary dihedral projective representations. If $\alpha^{\prime}$ is binary dihedral, the image of $\pi^{*}\left(\operatorname{ad} \alpha^{\prime}\right)=\operatorname{ad}\left(\pi^{*} \alpha^{\prime}\right): \pi_{1} \Sigma \rightarrow S O(3)$ belongs to $S O(2)$, which makes it a trivial representation. Therefore, ad $\alpha^{\prime}$ factors through $\pi_{1} \Sigma / \pi_{1} \Sigma^{\prime}=\mathbb{Z}_{n}$ and $\alpha^{\prime}$ cannot be irreducible.

The perturbation theory as described in Section 5 extends naturally to the case of projectively flat connections, see for instance [24, Section 5]. For a generic admissible perturbation $h$ of type (9), compare with [30, the perturbed projectively flat moduli space $\mathcal{R}_{w, h}^{*}\left(\Sigma^{\prime}, U(2)\right)$ is non-degenerate. In particular, it consists of finitely many points, and we define the Boyer-Nicas type invariant by the formula

$$
\lambda_{w}\left(\Sigma^{\prime}\right)=\frac{1}{2\left|H_{1}\left(\Sigma^{\prime} ; \mathbb{Z}_{2}\right)\right|} \sum_{\alpha^{\prime} \in \mathcal{R}_{w, h}^{*}\left(\Sigma^{\prime}, U(2)\right)}(-1)^{\mu\left(\alpha^{\prime}\right)},
$$

where $\mu\left(\alpha^{\prime}\right)$ is the spectral flow of the perturbed odd signature operator (44) along a generic path in $\mathcal{A}_{w}\left(\Sigma^{\prime}, U(2)\right)$ which connects a $U(2)$-lift of $\theta_{w}$ to a lift of $\alpha^{\prime}$.

According to Lemma [7.3, definitions (12) and (13) agree if $\mathcal{R}_{w}^{*}\left(\Sigma^{\prime}, S O(3)\right)$ is non-degenerate. Moreover, according to [24, Corollary 5.5], a small generic admissible perturbation $h$ which makes $\mathcal{R}_{w, h}^{*}\left(\Sigma^{\prime}, U(2)\right)$ non-degenerate can be chosen to be equivariant with respect to the action of $H^{1}\left(\Sigma^{\prime} ; \mathbb{Z}_{2}\right)$. If $h$ is small enough, this action still has no fixed points on $\mathcal{R}_{w, h}^{*}\left(\Sigma^{\prime}, U(2)\right)$. In addition, it preserves the spectral flow modulo 2, see [5, pages 239-240], so $\mu\left(\alpha^{\prime}\right)$ in (13) can be replaced by the spectral flow along a path of $S O(3)$-connections from $\theta_{w}$ to $\alpha^{\prime}$. This allows to view (13) as one half times the signed count of the perturbed flat $S O(3)$-connections.

Theorem 7.4 The invariants $\lambda_{w}\left(\Sigma^{\prime}\right)$ are well defined, and $\lambda_{0}\left(\Sigma^{\prime}\right)$ equals $n$ times the Boyer-Nicas invariant of $\Sigma$. Moreover, the sum of the $\lambda_{w}\left(\Sigma^{\prime}\right)$ over $w \in H^{2}\left(\Sigma^{\prime} ; \mathbb{Z}_{2}\right)$ equals the equivariant Casson invariant $\lambda^{\tau}(\Sigma)$.

Proof Since $\Sigma^{\prime}$ is cyclically finite, all reducible flat connections in the flat moduli space are isolated. This also remains true after a small admissible perturbation. Therefore, we can use the standard cobordism argument to show the 
well-definedness. Namely, for a generic path of small admissible perturbations $h(t), t \in[0,1]$, the parameterized moduli space

$$
\bigcup_{t \in[0,1]} \mathcal{R}_{w, h(t)}^{*}\left(\Sigma^{\prime}, U(2)\right) \times\{t\}
$$

gives a compact oriented cobordism between the perturbed flat moduli spaces $\mathcal{R}_{w, h(0)}^{*}\left(\Sigma^{\prime}, U(2)\right)$ and $\mathcal{R}_{w, h(1)}^{*}\left(\Sigma^{\prime}, U(2)\right)$. This allows us to conclude that the signed count of points in $\mathcal{R}_{w, h}^{*}\left(\Sigma^{\prime}, U(2)\right)$ is independent of $h$. A minor modification of Taubes' argument [30] and the above discussion of equivariant perturbations show that $\lambda_{0}\left(\Sigma^{\prime}\right)$ equals $n$ times the Boyer-Nicas invariant of $\Sigma$.

To prove the final statement, we use the identification (Proposition 7.1) between the moduli spaces $\mathcal{R}_{w}^{\tau}(\Sigma, S U(2))$ and $\mathcal{R}_{w}^{*}\left(\Sigma^{\prime}, S O(3)\right)$. If necessary, both spaces can be perturbed by using admissible perturbations (9) on $\Sigma^{\prime}$ and their pullbacks to $\Sigma$, so that the perturbed moduli spaces still match (the argument is completely similar to that in Section 3.8 of [7]). Note that, since $\Sigma^{\prime}$ is cyclically finite, the perturbations may be chosen so that they do not affect the reducible solutions.

Given a perturbed flat $S O(3)$-connection $\alpha^{\prime}$ in $P_{w}^{\prime}$, choose a generic path of connections $\alpha^{\prime}(t)$ connecting $\theta_{w}$ to $\alpha^{\prime}$. Lift it to a path $\pi^{*} \alpha^{\prime}(t)$ of $S U(2)-$ connections over $\Sigma$ equivariant with respect to a certain constant lift $u: P \rightarrow P$ and connecting $\theta$ to $\pi^{*} \alpha^{\prime}$. Then $\mu^{\tau}\left(\pi^{*} \alpha^{\prime}\right)=\mu\left(\alpha^{\prime}\right)$ due to the commutativity of the following diagram

$$
\begin{array}{cc}
\left(\Omega^{0} \oplus \Omega^{1}\right)_{u}(\Sigma, \operatorname{ad} P) \stackrel{K_{\pi^{*} \alpha^{\prime}(t)}^{u}}{\longrightarrow} & \left(\Omega^{0} \oplus \Omega^{1}\right)_{u}(\Sigma, \operatorname{ad} P) \\
\cong \uparrow \pi^{*} & \cong \uparrow \pi^{*} \\
\left(\Omega^{0} \oplus \Omega^{1}\right)\left(\Sigma^{\prime}, \text { ad } P_{w}^{\prime}\right) \stackrel{K_{\alpha^{\prime}(t)}}{\longrightarrow} & \left(\Omega^{0} \oplus \Omega^{1}\right)\left(\Sigma, \operatorname{ad} P_{w}^{\prime}\right) .
\end{array}
$$

Remark 7.5 According to Theorem 7.4 no $H^{1}\left(\Sigma^{\prime} ; \mathbb{Z}_{2}\right)$-equivariant perturbation is needed to compute $\lambda_{w}\left(\Sigma^{\prime}\right)$ using definition (13) - any sufficiently small generic admissible perturbation $h$ which makes $\mathcal{R}_{w, h}^{*}\left(\Sigma^{\prime}, U(2)\right)$ non-degenerate will do.

\subsection{The formula}

Recall first that any homology lens space $\Sigma^{\prime}$ such that $H_{1}\left(\Sigma^{\prime} ; \mathbb{Z}\right)=\mathbb{Z}_{n}$ can be obtained by $(n / q)$-surgery along a knot $k$ in an integral homology sphere $Y$, where $q$ is relatively prime to $n$ and $0<q<n$, see for instance [4]. 
Proposition 7.6 Suppose that $\Sigma^{\prime}=Y+(n / q) \cdot k$. Denote by $\lambda(Y)$ the (regular) Casson invariant of $Y$, and by $\Delta(t)$ the Alexander polynomial of $k \subset Y$ normalized so that $\Delta(1)=1$ and $\Delta(t)=\Delta\left(t^{-1}\right)$. Then, if $n$ is even,

$$
\lambda_{w}\left(\Sigma^{\prime}\right)=\frac{n}{2} \lambda(Y)+\frac{1}{8} \sum_{\substack{m=0 \\ m=w(\bmod 2)}}^{n-1} \operatorname{sign}^{m / n} k+\frac{q}{4} \Delta^{\prime \prime}(1), \quad w=0,1,
$$

and, if $n$ is odd,

$$
\lambda_{0}\left(\Sigma^{\prime}\right)=n \lambda(Y)+\frac{1}{8} \sum_{m=0}^{n-1} \operatorname{sign}^{m / n} k+\frac{q}{2} \Delta^{\prime \prime}(1) .
$$

By $\operatorname{sign}^{\alpha} k$ we mean the Tristram-Levine equivariant knot signature, see Section 6. The following result is immediate from Theorem 7.4 and Proposition 7.6 .

Corollary 7.7 For any $n \geq 1$, the equivariant Casson invariant can be expressed by the formula

$$
\lambda^{\tau}(\Sigma)=n \lambda(Y)+\frac{1}{8} \sum_{m=0}^{n-1} \operatorname{sign}^{m / n} k+\frac{q}{2} \Delta^{\prime \prime}(1) .
$$

Remark 7.8 Boyer and Lines 4] inquired whether the invariant of the homology lens space $\Sigma^{\prime}=Y+(n / q) \cdot k$ defined by the formula

$$
\bar{\lambda}\left(\Sigma^{\prime}\right)=\lambda(Y)+\frac{1}{8 n} \sum_{m=0}^{n-1} \operatorname{sign}^{m / n} k+\frac{q}{2 n} \Delta^{\prime \prime}(1)
$$

coincides with the Boyer-Nicas invariant $(1 / n) \cdot \lambda_{0}\left(\Sigma^{\prime}\right)$, see Question 2.24 of 4]. Proposition 7.6 implies that indeed $n \cdot \bar{\lambda}\left(\Sigma^{\prime}\right)=\lambda_{0}\left(\Sigma^{\prime}\right)$ if $n$ is odd; however, $n \cdot \bar{\lambda}\left(\Sigma^{\prime}\right)=\lambda_{0}\left(\Sigma^{\prime}\right)+\lambda_{1}\left(\Sigma^{\prime}\right)$ if $n$ is even.

The rest of this section is devoted to the proof of Proposition [7.6

\subsection{Small scale perturbations}

Let $X=Y \backslash N(k)$ be the knot $k$ exterior, and $m$ and $\ell$ be the canonical meridian and longitude on $\partial X$. Let $\mathcal{P}=\mathcal{R}(\partial X, S U(2))$ be the $S U(2)$ pillowcase with coordinates $(\varphi, \psi)$ such that the holonomies along $m$ and $\ell$ equal $\exp (i \varphi)$ and $\exp (i \psi)$, respectively, shown in Figure 1. The inclusion $\partial X \rightarrow X$ induces a natural restriction map $r: \mathcal{R}(X, S U(2)) \rightarrow \mathcal{P}$ whose image is generically one-dimensional. 


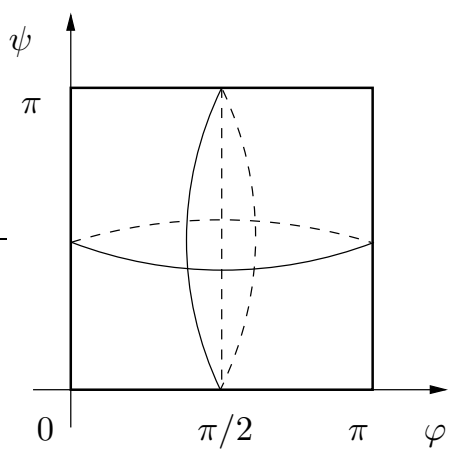

Figure 1

Technically, it is more convenient to work with double covers of the above as in [15]. More precisely, if we fix the maximal torus $U(1)=\{\exp (i \varphi)\} \subset S U(2)$ then the space of flat $U(1)$-connections on $\partial X$ modulo $U(1)$-gauge group is a natural double cover $\tilde{\mathcal{P}}=T^{2}$ of $\mathcal{P}$ branched at the central orbits. It gives rise to the double cover $\tilde{\mathcal{R}}(X, S U(2))$ and the restriction map $r: \tilde{\mathcal{R}}(X, S U(2)) \rightarrow \tilde{\mathcal{P}}$. The 2-torus $\tilde{\mathcal{P}}$ will be depicted as the square $\tilde{\mathcal{P}}=\{(\varphi, \psi) \mid 0 \leq \varphi \leq 2 \pi, 0 \leq$ $\psi \leq 2 \pi\}$ with properly identified sides; the double covering $\tilde{\mathcal{P}} \rightarrow \mathcal{P}$ then corresponds to the quotient map by the action $\sigma(\varphi, \psi)=(2 \pi-\varphi, 2 \pi-\psi)$ (which is geometrically a $180^{\circ}$ rotation of the square $\tilde{\mathcal{P}}$ about the point $(\pi, \pi)$ ). The image of $r: \tilde{\mathcal{R}}(X, S U(2)) \rightarrow \tilde{\mathcal{P}}$ is invariant with respect to this action. For instance, if $k$ is the left-handed trefoil, the image of $\tilde{\mathcal{R}}(X, S U(2))$ in $\tilde{\mathcal{P}}$ consists of the circle $\{\psi=0\}$ and two open arcs limiting to it, as shown in bold in Figure 2

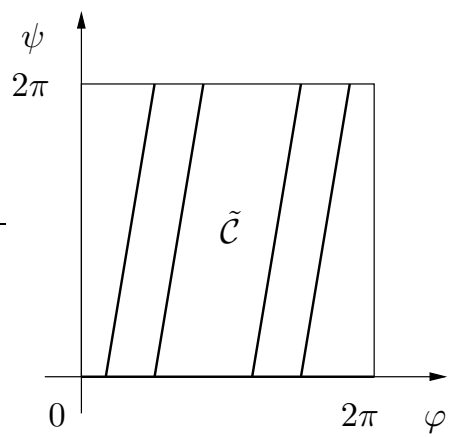

Figure 2

We will perturb the flat moduli space if necessary using admissible perturbations $h$ described in [16] (as amended in [17]). The moduli space of $h$-perturbed 
flat connections will be denoted by $\mathcal{R}_{h}(X, S U(2))$, and its double cover by $\tilde{\mathcal{R}}_{h}(X, S U(2))$.

According to [16], for a generic admissible perturbation $h$, the perturbed flat moduli space $\tilde{\mathcal{R}}_{h}(X, S U(2))$ consists of two central orbits, two smooth open arcs of abelian orbits with one non-compact end limiting to each central orbit (together with the central orbits, they form a circle), and a smooth 1-dimensional manifold of irreducible orbits with finitely many ends, each limiting to a different point on the abelian arc. If a point on the abelian arc with coordinate $\varphi$ is the limiting point of an irreducible arc then $\exp (2 i \varphi)$ is a root of $\Delta(t)$. Since (see Section 8) the $n$-fold cyclic branched cover of $k \subset Y$ is an integral homology sphere, $\Delta(\exp (2 \pi i m / n)) \neq 0$ for $m \in \mathbb{Z}$, and we can conclude that

(a) no irreducible arc limits to a point on the abelian arc with coordinate $\varphi=\pi m / n$.

Furthermore, the restriction map $r: \tilde{\mathcal{R}}_{h}(X, S U(2)) \rightarrow \tilde{\mathcal{P}}$, which is well defined because $h$ is supported away from $N(k)$, is an immersion taking the 1dimensional strata of $\tilde{\mathcal{R}}_{h}(X, S U(2))$ into the part of $\tilde{\mathcal{P}}$ away from the branching points. The map $r$ restricted to the circle of reducible orbits is an embedding with image $\{\psi=0\}$. The image of the irreducible part of $\tilde{\mathcal{R}}_{h}(X, S U(2))$ in $\tilde{\mathcal{P}}$ will be denoted by $\tilde{\mathcal{C}}$ (compare with Figure 2). By using another small admissible perturbation if necessary, one can ensure the following:

(b) the intersections of $\tilde{\mathcal{C}}$ with all of the circles $\mathcal{S}(\pi m / n) \subset \tilde{\mathcal{P}}$ given by the equation $\varphi=\pi m / n, 0 \leq m \leq 2 n$, are transverse (see [15], Lemma 5.1);

(c) the intersection of $\tilde{\mathcal{C}}$ with the circle $\psi=\pi$ is transverse, and the $\varphi-$ coordinates of the intersection points are different from $\pi m / n, 0 \leq m \leq$ $2 n$. This can be achieved by additional perturbations on loops $\gamma$ which are contained in a collar neighborhood of $\partial X$ and have the property that $\operatorname{lk}(\gamma, k) \neq 0$ (see [16], page 408);

(d) the intersections of $\tilde{\mathcal{C}}$ with the curve $\psi=-(n / q) \cdot \varphi$, and also with the curve $\psi=\pi-(n / q) \cdot \varphi$ if $n$ is even, are transversal (using the loops $\gamma$ as in (c) again). These intersections correspond to the respective $\mathcal{R}_{w, h}^{*}\left(\Sigma^{\prime}, U(2)\right)$, as we will explain below.

Note that, after all the admissible perturbations, $\tilde{\mathcal{C}}$ remains invariant with respect to the involution $\sigma$. 


\subsection{Large scale perturbations}

Recall that $\Sigma^{\prime}$ is given by $(n / q)$ Dehn surgery along a knot $k \subset Y$, ie, by adding a solid torus to $X$. Parameterize this solid torus by $\gamma: S^{1} \times D^{2} \rightarrow \Sigma^{\prime}$, and write $k^{\prime}$ for its core $S^{1} \times\{0\}$. Let $f: S U(2) \rightarrow \mathbb{R}$ be a smooth function which is invariant with respect to conjugation. Such a function is determined by a function $\tilde{f}:[0, \pi] \rightarrow \mathbb{R}$ by the formula $\tilde{f}(t)=f(\exp (i t))$. Note that the smoothness of $f$ requires that $\tilde{f}^{\prime}$ vanish at $t=0$ and $t=\pi$. We will extend $\tilde{f}$ to a function on $[0,2 \pi]$ by the rule $\tilde{f}(t)=\tilde{f}(2 \pi-t)$ for $\pi \leq t \leq 2 \pi$. Let $h_{0}$ be the admissible perturbation given by the formula (compare with (9))

$$
h_{0}(\alpha)=\int_{D^{2}} f\left(\operatorname{hol}_{\alpha}\left(\gamma\left(S^{1} \times\{z\}\right), s\right)\right) \eta(z) d^{2} z .
$$

Notice that the knot exterior $X$ is naturally a subset of $\Sigma^{\prime}$. Any perturbed flat connection over $\partial X$ is actually flat hence it is determined by two parameters $a, b$ where, in a suitable parametrization, $\exp (i a)$ is the holonomy around meridian (the boundary of the disc $D^{2}$ ) and $\exp (i b)$ is the holonomy around the longitude (a curve parallel to $k$ ). The following is a minor modification of Lemma 4 of $[5$.

Lemma 7.9 For sufficiently small $\varepsilon>0$, restriction to $X$ defines a one-to-one correspondence between

(a) the gauge equivalence classes of $\left(h_{0}+\varepsilon h\right)$-perturbed flat connections on $\Sigma^{\prime}$, and

(b) the gauge equivalence classes of $\varepsilon h$-perturbed flat connections on $X$ satisfying the equation $a=\tilde{f}^{\prime}(b)$.

The extension condition (b) in Lemma 7.9 (ie, the equation $\left.a=\tilde{f}^{\prime}(b)\right)$ defines a curve in $\tilde{\mathcal{P}}$. In the case that $f \equiv 0$, this curve would be the line $\psi=-(n / q) \cdot \varphi$, using coordinates $(\varphi, \psi)$ dual to the meridian and longitude of $k$ (which differ from those of $k^{\prime}$ ). A more realistic example may look as shown in Figure 3 The figure shows, for $(n / q)=(2 / 1)$, the curves $\psi=-(n / q) \cdot \varphi$ and $a=\tilde{f}^{\prime}(b)$. Note that the curve $a=\tilde{f}^{\prime}(b)$ is invariant with respect to $\sigma$.

\subsection{The case of even $n$}

Let us place $\tilde{\mathcal{C}}$ in general position as in Section [7.4 then $\mathcal{R}_{0, h}^{*}\left(\Sigma^{\prime}, U(2)\right)$ is in one-to-one correspondence with the orbits of $\sigma$ acting on $\tilde{\mathcal{C}} \cap\{\psi=-(n / q) \cdot \varphi\}$. 


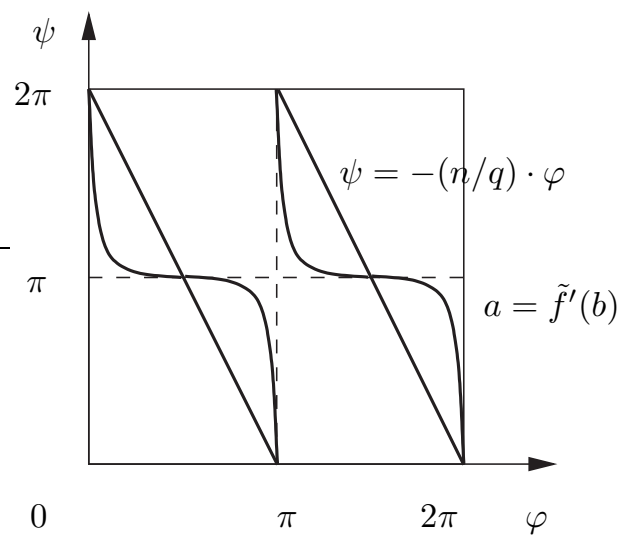

Figure $3: n=2, q=1$

Note that this action is free, see Lemma 7.3 so each such orbit has two elements. Taking into account the $1 / 4$ factor in the definition (13) and the orientations set in Section 7.8 the invariant $\lambda_{0}\left(\Sigma^{\prime}\right)$ equals one eighth times the intersection number of $\tilde{\mathcal{C}}$ with the curve $\psi=-(n / q) \cdot \varphi$. This can be proved along the same lines as [15, Theorem 7.1]. The heart of that proof is a spectral flow calculation that does not require that $\Sigma^{\prime}$ be an integral homology sphere.

The curve $\psi=-(n / q) \cdot \varphi$ wraps $n$ times around $\tilde{\mathcal{P}}$ in the $\psi$-direction, and the points of intersection with the curve $\psi=0$ separate it into $n$ segments given by $2 \pi j q / n \leq \varphi \leq 2 \pi(j+1) q / n$ where $j=0, \ldots, n-1$. By choosing a suitable function $f$, one can deform each of these segments in a $\sigma$-equivariant manner into a graph $a=\tilde{f}^{\prime}(b)$ which lies arbitrarily close to the union

$$
\tilde{S}(2 \pi j q / n) \cup \tilde{S}(2 \pi(j+1) q / n) \cup\{\psi=\pi\},
$$

as shown in Figure 3. With orientations set as in Section 7.8, we see that the intersection number of $\tilde{\mathcal{C}}$ with the curve $\psi=-(n / q) \cdot \varphi$ is the sum of two terms. The first one is the intersection number of $\tilde{\mathcal{C}}$ with the union of the circles $\tilde{S}(2 \pi j / n)$ over $j=0, \ldots, n-1$. According to [15], it equals

$$
4 n \cdot \lambda(Y)+\frac{1}{2} \sum_{j=0}^{n-1} \operatorname{sign}^{2 j / n} k=4 n \cdot \lambda(Y)+\sum_{\substack{m=0 \\ m \text { even }}}^{n-1} \operatorname{sign}^{m / n} k .
$$

The second term equals $q$ times the intersection number of $\tilde{\mathcal{C}}$ with the circle $\{\psi=\pi\}$; this is given by $-2 \Delta^{\prime \prime}(1)$. Up to an overall sign, this can be seen as in [15. Proposition 7.2] using the gauge theoretic description of $\Delta^{\prime \prime}(1)$ given 
in 19]; the sign is then fixed with the help of the Casson surgery formula $\lambda(Y-k)=\lambda(Y)-(1 / 2) \Delta^{\prime \prime}(1)$. This proves the formula for $\lambda_{0}\left(\Sigma^{\prime}\right)$.

To prove the formula for $\lambda_{1}\left(\Sigma^{\prime}\right)$, we consider the intersection $\tilde{\mathcal{C}} \cap\{\psi=\pi-$ $(n / q) \cdot \varphi\}$ and deform the curve $\psi=\pi-(n / q) \cdot \varphi$ again as shown in Figure 4. The rest of the proof follows the lines of the above proof for $\lambda_{0}\left(\Sigma^{\prime}\right)$. To express $\lambda_{1}\left(\Sigma^{\prime}\right)$ as the intersection number in $\tilde{\mathcal{P}}$, we again refer to the proof of 15. Theorem 7.1], where the trivial connection should be replaced with $\theta_{1}$, see Figure 4

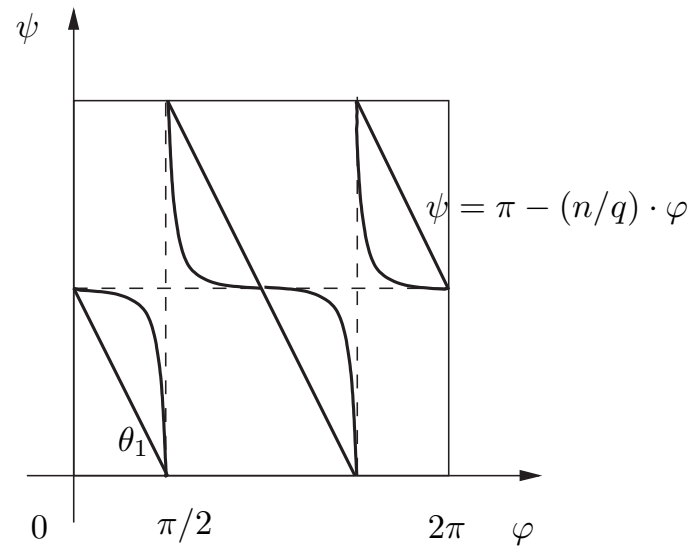

Figure $4: n=2, q=1$

\subsection{The case of odd $n$}

The proof is completely similar to that for $\lambda_{0}\left(\Sigma^{\prime}\right)$ in the even case. The invariant $\lambda_{0}\left(\Sigma^{\prime}\right)$ equals one fourth times the intersection number of $\tilde{\mathcal{C}}$ with the curve $\psi=-(n / q) \cdot \varphi$ (after fixing orientations). Deforming $\psi=-(n / q) \cdot \varphi$ as shown in Figure 3] we see that the above intersection number equals the sum of two terms. The first one is the intersection number of $\tilde{\mathcal{C}}$ with the union of the circles $\tilde{S}(2 \pi j / n)$ over $j=0, \ldots, n-1$. According to [15], it equals

$$
4 n \cdot \lambda(Y)+\frac{1}{2} \sum_{j=0}^{n-1} \operatorname{sign}^{2 j / n} k=4 n \cdot \lambda(Y)+\frac{1}{2} \sum_{m=0}^{n-1} \operatorname{sign}^{m / n} k
$$

(we used the fact that $\operatorname{sign}^{\alpha} k=\operatorname{sign}^{1+\alpha} k$ ). The second term as before equals $q$ times the intersection number of $\tilde{\mathcal{C}}$ with the circle $\{\psi=\pi\}$, which equals $-2 \Delta^{\prime \prime}(1)$. This completes the proof. 


\subsection{Orientations}

The tangent space of $\tilde{\mathcal{P}}$ at any point is canonically isomorphic to $H^{1}(\partial X ; \mathbb{R})$ and hence is canonically oriented after we orient $\partial X$ as the boundary of $X$. We adapt the convention that an outward normal vector followed by an oriented basis for $T_{x}(\partial X)$ gives an oriented basis for $T_{x} X$ (this is opposite to the convention used in [15]).

Orientations of various curves in the above construction depend on an orientation of $H^{1}(X ; \mathbb{R})$ or, equivalently, a choice of meridian. Once it is fixed, the circles $\{\psi=0\}$ and $\{\psi=\pi\}$ are naturally oriented by the parameter $\varphi$. The circles $S(\alpha)$ are oriented by the requirement that the intersection number of $\{\psi=0\}$ with $S(\alpha)$ be one; the same requirement orients the curves $\{\psi=-(n / q) \cdot \varphi\}$ and $\{\psi=\pi-(n / q) \cdot \varphi\}$. The curve $\tilde{\mathcal{C}}$ is oriented as described in [15], Section 2.

Note that $\sigma$ reverses the orientation of $H^{1}(X ; \mathbb{R})$; however, it preserves all the intersection numbers.

\section{$7.9 \quad$ Examples}

Let $p, q$, and $r$ be pairwise relatively prime positive integers, and consider the Brieskorn homology sphere

$$
\Sigma(p, q, r)=\left\{x^{p}+y^{q}+z^{r}=0\right\} \cap S^{5} \subset \mathbb{C}^{3} .
$$

The map $\tau(x, y, z)=\left(x, y, e^{2 \pi i / r} z\right)$ defines a $\mathbb{Z}_{r}$-action on $\Sigma(p, q, r)$ whose fixed point set is the singular fiber $k_{r}=\Sigma(p, q, r) \cap\{z=0\}$. The quotient of $\Sigma(p, q, r)$ by this action is $S^{3}$, with branch set the right-handed $(p, q)$-torus knot $k$. In particular, if we write $\Sigma(p, q, r)=X \cup N\left(k_{r}\right)$ where $X$ is the knot exterior, then the action on $N\left(k_{r}\right)=S^{1} \times D^{2}$ will be given by the formula $(s, z) \mapsto\left(s, e^{2 \pi i / r} z\right)$.

The Brieskorn homology sphere $\Sigma(p, q, r+p q)$, obtained from $\Sigma(p, q, r)$ by $(-1)$-surgery along $k_{r}$, admits a free $\mathbb{Z}_{r}$-action which extends the action on $X$ to $S^{1} \times D^{2}$ by the formula $(s, z) \mapsto\left(e^{2 \pi i / r} s, e^{2 \pi i / r} z\right)$. If $\Sigma(p, q, r+p q)$ is viewed as a link of singularity, this action is given by

$$
\tau(x, y, z)=\left(e^{2 \pi i q / r} x, e^{2 \pi i p / r} y, e^{2 \pi i / r} z\right) .
$$

Corollary 7.7 can be used to compute the equivariant Casson invariant of $\Sigma(p, q, r+p q)$ with respect to the action (16) as follows. 
Let $\Sigma^{\prime}$ be the quotient manifold of $\Sigma(p, q, r+p q)$. It is immediate from the above description that $\Sigma^{\prime}$ can be obtained from $S^{3}$ by $(-r)$-surgery along the $(p, q)$-torus knot $k$. Therefore,

$$
\lambda^{\tau}(\Sigma(p, q, r+p q))=\frac{1}{8} \sum_{m=0}^{r-1} \operatorname{sign}^{m / r} k-\frac{1}{2} \Delta_{k}^{\prime \prime}(1),
$$

where

$$
\begin{aligned}
\frac{1}{8} \sum_{m=0}^{r-1} \operatorname{sign}^{m / r} k & =\lambda^{\tau}(\Sigma(p, q, r)), \quad \text { by (11), } \\
& =\lambda(\Sigma(p, q, r)), \quad \text { according to [7], }
\end{aligned}
$$

and $\Delta_{k}^{\prime \prime}(1)=\Delta_{k_{r}}^{\prime \prime}(1)=\left(p^{2}-1\right)\left(q^{2}-1\right) / 12$, see [20]. Now, the right hand side of (17) equals $\lambda(\Sigma(p, q, r))-(1 / 2) \Delta_{k_{r}}^{\prime \prime}(1)$, which coincides with $\lambda(\Sigma(p, q, r+p q))$ by Casson's surgery formula. Therefore,

$$
\lambda^{\tau}(\Sigma(p, q, r+p q))=\lambda(\Sigma(p, q, r+p q)) .
$$

Another way to see this would be using the fact that $\tau$ can be included into the natural $S^{1}$-action on the link $\Sigma(p, q, r+p q)$ given by

$$
(x, y, z) \mapsto\left(t^{q(r+p q)} x, t^{p(r+p q)} y, t^{p q} z\right)
$$

and is therefore isotopic to the identity.

\section{The Rohlin invariant}

Our next goal is prove that $\lambda^{\tau}(\Sigma)$ equals the Rohlin invariant $\rho(\Sigma)$ modulo 2, and thus verify Theorem 1.2. This is a known fact when $\operatorname{Fix}(\tau) \neq \emptyset$, see [7. In the case of $\operatorname{Fix}(\tau)=\emptyset$, we will achieve our goal by identifying the right hand side of (14) with $\rho(\Sigma)$ modulo 2 .

\subsection{Reduction to the Arf-invariants}

We use notations of Section 7.3 Let $Y_{n}$ be the $n$-fold branched cover of $Y$ with branch set the knot $k$; the preimage of $k$ in $Y_{n}$ is a knot which we call $k_{n}$. Observe that $\Sigma=Y_{n}+(1 / q) \cdot k_{n}$ so that $Y_{n}$ is an integral homology sphere. According to (11),

$$
n \cdot \lambda(Y)+\frac{1}{8} \sum_{m=0}^{n-1} \operatorname{sign}^{m / n} k=\lambda^{\tau}\left(Y_{n}\right)
$$


is the equivariant Casson invariant of $Y_{n}$, whose modulo 2 reduction is known 7 , Theorem 2] to equal $\rho\left(Y_{n}\right)$. Since $(1 / 2) \Delta^{\prime \prime}(1)=\operatorname{arf}(k)(\bmod 2)$, we only need to show that $\rho(\Sigma)=\rho\left(Y_{n}\right)+q \cdot \operatorname{arf}(k)(\bmod 2)$. Now, $\rho(\Sigma)=\rho\left(Y_{n}\right)+q \cdot \operatorname{arf}\left(k_{n}\right)$ (mod 2) by Rohlin's surgery formula. Therefore, the proof will be complete after we show the following.

Proposition 8.1 Let $Y$ be an integral homology sphere and $\pi: Y_{n} \rightarrow Y$ be its $n$-fold cyclic branched cover with branch set a knot $k$. Let $k_{n}$ be the knot $\pi^{-1}(k)$ in $Y_{n}$. If $Y_{n}$ is an integral homology sphere then $\operatorname{arf}\left(k_{n}\right)=\operatorname{arf}(k)$ $(\bmod 2)$.

\subsection{An algebraic lemma}

Proposition 8.1 will be deduced from a purely algebraic lemma, using Levine's theorem [18] connecting the Arf invariant and the Alexander polynomial. A polynomial $\Delta(t) \in \mathbb{Z}\left[t, t^{-1}\right]$ is said to be symmetric and normalized if $\Delta(t)=$ $\Delta\left(t^{-1}\right)$ and $\Delta(1)=1$. Note that this implies that $\Delta(-1) \equiv 1$ or $5(\bmod 8)$. Levine showed that if the Alexander polynomial of a knot is symmetric and normalized, the Arf invariant of the knot is 0 if $\Delta(-1) \equiv 1(\bmod 8)$ and is 1 if $\Delta(-1) \equiv 5(\bmod 8)$.

Lemma 8.2 Suppose that $\Delta(t), D(t) \in \mathbb{Z}\left[t, t^{-1}\right]$ are symmetric, normalized polynomials. For an integer $n$, denote by $\omega$ a primitive $n^{\text {th }}$ root of unity. Assume that $D\left(t^{n}\right)=\prod_{j=0}^{n-1} \Delta\left(\omega^{j} t\right)$. If $n$ is even, then $D(-1)=\Delta(-1) \equiv 1$ $(\bmod 8)$. If $n$ is odd, then $D(-1)=a \cdot \Delta(-1)$ where $a \in \mathbb{Z}$ is congruent to 1 $(\bmod 8)$.

Proof Write $n=2 p+1$ if $n$ is odd, and $n=2 p$ if $n$ is even. If $\xi$ is a primitive $2 n^{\text {th }}$ root of unity, then $\xi^{n}=-1$. Write $\nu=\Delta(\xi) \Delta\left(\xi^{3}\right) \cdots \Delta\left(\xi^{2 p-1}\right)$. Note that the symmetry of $\Delta$ means that $\Delta\left(\xi^{k}\right)=\Delta\left(\xi^{2 n-k}\right)$. If $n=2 p$, this means that the last term in $\nu$ is $\Delta\left(\xi^{4 p-2 p+1}\right)=\Delta\left(\xi^{2 p+1}\right)$. Likewise, if $n=2 p+1$, then the last term is $\Delta\left(\xi^{2 p+3}\right)$. Now compute

$$
\begin{aligned}
D(-1) & =\prod_{j=0}^{n-1} \Delta\left(\xi^{2 j+1}\right)=\Delta(\xi) \Delta\left(\xi^{3}\right) \cdots \Delta\left(\xi^{2 p-1}\right) \Delta\left(\xi^{2 p+1}\right) \cdots \Delta\left(\xi^{2 n-1}\right) \\
& = \begin{cases}\nu^{2}, & n \text { even, } \\
\nu^{2} \Delta\left(\xi^{2 p+1}\right), & n \text { odd } .\end{cases}
\end{aligned}
$$


Now $D(-1)$, being congruent to $D(1)(\bmod 2)$ is odd, as is $\Delta(-1)$. We claim that $\nu$, which is a priori an algebraic integer, is actually an integer. In the case that $n=2 p+1$ is odd, $\Delta\left(\xi^{2 p+1}\right)=\Delta(-1)$, so $\nu^{2}$ will necessarily be the square of an odd integer, and therefore congruent to $1(\bmod 8)$. In the case that $n$ is even, we get by the same argument that $D(-1)$ is congruent to $1(\bmod 8)$.

To see that $\nu$ is an integer, we use a little Galois theory. By definition, $\nu \in \mathbb{Z}(\xi)$, which is the ring of integers in the cyclotomic field $\mathbb{Q}(\xi)$. We need to show that $\nu$ is fixed by every element of the Galois group of the field extension $\mathbb{Q}(\xi) / \mathbb{Q}$. This group is the (multiplicative) group of units $\mathbb{Z}_{2 n}^{\times}$, where $r \in \mathbb{Z}_{2 n}^{\times}$acts by $\xi^{j} \rightarrow \xi^{j r}$. Note that the set of odd numbers $\{1,3, \ldots, 2 n-1\} \subset \mathbb{Z}_{2 n}$ is invariant under $j \rightarrow-j(\bmod 2 n)$. If $n$ is even, the orbits all have two elements, one $\leq 2 p-1$ and one $\geq 2 p+1$. If $n$ is odd, there is one orbit, namely $2 p+1$, with one element while all the others have two elements. Consider the action of $r \in \mathbb{Z}_{2 n}^{\times}$on the exponents appearing in $\nu$, namely $E:=\{1,3, \ldots, 2 p-1\}$; note that $r$ preserves orbits since $r(-a)=-r a$. Now if $r a \in E$ then $-r a \notin E$, and so $r E$ consists of one element of each orbit (except $2 p+1$ if $n$ is odd). It follows that $r \cdot \nu=r \cdot \prod_{a \in E} \Delta\left(\xi^{a}\right)=\prod_{a \in E} \Delta\left(\xi^{r a}\right)=\prod_{a \in E} \Delta\left(\xi^{ \pm a}\right)=\nu$.

For odd $n$, we are finished, but for $n$ even, we still have to show that $\Delta(-1) \equiv 1$ $(\bmod 8)$. In fact, $\Delta(-1)=1$, by the following argument :

$$
\begin{aligned}
D(1) & =\prod_{j=0}^{n-1} \Delta\left(\omega^{j+1}\right) \\
& =\Delta(1) \Delta\left(\omega^{p}\right) \Delta(\omega) \Delta\left(\omega^{2}\right) \cdots \Delta\left(\omega^{p-1}\right) \Delta\left(\omega^{p+1}\right) \cdots \Delta\left(\omega^{n-1}\right) \\
& =\Delta(1) \Delta(-1) \eta
\end{aligned}
$$

for some $\eta$. Now the Galois group of $\mathbb{Q}(\omega) / \mathbb{Q}$ permutes the elements $\omega^{j}$, and no element $\omega^{j}$ for $j \neq p$ gets sent to -1 . So the Galois group preserves $\eta$. Hence $\eta \in \mathbb{Z}$, so $\Delta(-1) \mid D(1)$. Since $D(1)=1$ and $\Delta$ is symmetric and normalized, it follows that $\Delta(-1)=1$.

Remark 8.3 The final argument is clearly equivalent to (and is inspired by) the fact that there is a branched cover $Y_{2 p} \rightarrow Y_{2}$, so the hypothesis that $Y_{n}$ be a homology sphere implies that $Y_{2}$ is one also.

\subsection{Proof of Proposition 8.1}

If $k$ is a knot in the homology sphere $Y$, and the $n$-fold cyclic branched cover $Y_{n}$ is a homology sphere, then the Alexander polynomials of $k$ and $k_{n}$ are related 
as in Lemma 8.2, see [1]. Levine's theorem implies that their Arf invariants must be the same.

Remark 8.4 Chuck Livingston has pointed out a more geometric proof of Proposition 8.1 that has the additional advantage of applying to $\mathbb{Z}_{2}$ homology spheres. Here is an outline of the argument. Livingston observes that the Arf invariant of a knot in a $\mathbb{Z}_{2}$ homology sphere $Y$ can be defined as the spinbordism class of a Seifert surface for the knot, using the unique spin structure induced from $Y$. This spin structure, restricted to the knot complement, pulls back under the branched covering, and clearly gives the same spin structure on a lift of the Seifert surface. By obstruction theory, if the branched cover is also a a $\mathbb{Z}_{2}$ homology sphere, this lifted spin structure (or one that is identical in a neighborhood of the lifted Seifert surface) can be extended over the branched covering. Hence the Arf invariant upstairs is the same as the Arf invariant downstairs.

\section{Applications}

The relations between the equivariant Casson invariant on one hand, and Donaldson polynomials and Floer Lefschetz numbers, on the other, lead to several interesting applications which we describe in this section.

\subsection{Invariant $\lambda^{\tau}(\Sigma)$ as a function of $\tau$}

Let $\Sigma$ be an oriented integral homology sphere, and $\tau: \Sigma \rightarrow \Sigma$ be an orientation preserving diffeomorphism of finite order. Theorem 1.1 identifies $\lambda^{\tau}(\Sigma)$ with one fourth of the degree-zero Donaldson polynomial of the mapping torus of $\tau$.

If $\tau$ is (smoothly) isotopic to the identity, then the mapping torus of $\tau$ is diffeomorphic to the product $S^{1} \times \Sigma$. Since the degree-zero Donaldson polynomial is an invariant of diffeomorphism, we conclude that $\lambda^{\tau}(\Sigma)=\lambda(\Sigma)$. It is not clear if this result can be obtained directly from the definition of $\lambda^{\tau}(\Sigma)$. In the case that $\tau$ is contained in a circle action, this was shown by direct calculation in [7].

\subsection{Plumbed manifolds}

The equivariant Casson approach to the Floer Lefschetz number allows one to actually compute that number in several interesting examples. 
Given a plumbed homology sphere $\Sigma$, there is a preferred class of orientation preserving involutions $\tau$ which represent $\Sigma$ as a double branched cover of $S^{3}$ with branch set a Montesinos knot $k_{\tau}$, see [29]. The knots corresponding to different involutions from this class are related by a sequence of mutations. Since the knot signature is a mutation invariant, this construction ends up in a well defined integer valued invariant $\bar{\mu}(\Sigma)=(1 / 8) \cdot \operatorname{sign}\left(k_{\tau}\right)$ called the $\bar{\mu}$-invariant, see [21] and [29]. It is immediate from (11) that $\bar{\mu}(\Sigma)=\lambda^{\tau}(\Sigma)$.

More can be said if $\Sigma$ is a Seifert fibered homology sphere $\Sigma\left(a_{1}, \ldots, a_{n}\right)$. In this case, $\tau$ is induced by complex conjugation on $\Sigma\left(a_{1}, \ldots, a_{n}\right)$ viewed as a link of complex singularity. According to [27], there exists an equivariant admissible perturbation $h$ such that assumption $(*)$ of Section 3.7 holds (whether the same holds for arbitrary plumbed homology spheres remains to be seen). Therefore, we have the following result.

Proposition 9.1 The $\bar{\mu}$-invariant of a Seifert homology sphere $\Sigma\left(a_{1}, \ldots, a_{n}\right)$ equals half the Lefschetz number of $\tau_{*}: I_{*}\left(\Sigma\left(a_{1}, \ldots, a_{n}\right)\right) \rightarrow I_{*}\left(\Sigma\left(a_{1}, \ldots, a_{n}\right)\right)$.

According to [10], the group $I_{k}\left(\Sigma\left(a_{1}, \ldots, a_{n}\right)\right)$ is trivial if $k$ is even, and is free abelian group of rank, say, $b_{k}$ if $k$ is odd.

Proposition 9.2 The map $\tau_{*}: I_{k}\left(\Sigma\left(a_{1}, \ldots, a_{n}\right)\right) \rightarrow I_{k}\left(\Sigma\left(a_{1}, \ldots, a_{n}\right)\right)$ is identity if $k=1(\bmod 4)$ and minus identity if $k=3(\bmod 4)$. In particular, the Floer Lefschetz number of $\tau_{*}$ equals $-b_{1}+b_{3}-b_{5}+b_{7}$.

Proof The generators $\alpha$ of the Floer chain complex of $\Sigma\left(a_{1}, \ldots, a_{n}\right)$ can be chosen so that all of them have odd Floer index and are preserved by the action induced by $\tau$, see [27. According to [26] and 27, the equivariant spectral flow for these generators is given by the formula $\mu^{\tau}(\alpha)=\frac{1}{2}(\mu(\alpha)+1)(\bmod 4)$. The result is now immediate from the following calculation, compare with Section 3.7:

$$
\tau_{*}(\alpha)=(-1)^{\mu^{\tau}(\alpha)-\mu(\alpha)} \alpha=(-1)^{(k-1) / 2} \alpha .
$$

\subsection{Akbulut cork}

Another situation when the Floer Lefschetz number can be computed explicitly is that of the Akbulut cork. By Akbulut cork we mean the smooth contractible 4-manifold $W$ obtained by attaching a two-handle to $S^{1} \times D^{3}$ along its boundary as shown in Figure 5. It can be embedded into a blown up elliptic surface $E(n) \#\left(-\mathbb{C} P^{2}\right)$ in such a way that cutting it out and re-gluing by an involution 


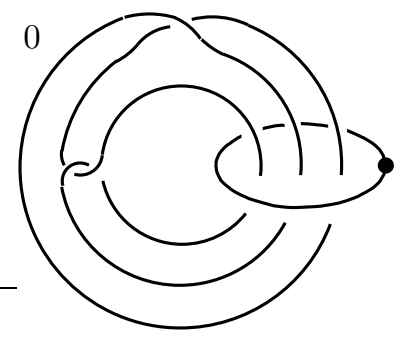

Figure 5

on $\Sigma=\partial W$ changes the smooth structure on $E(n) \#\left(-\mathbb{C} P^{2}\right)$ but preserves its homeomorphism type, see [1] and [14].

The involution $\tau: \Sigma \rightarrow \Sigma$ simply interchanges the two link components, $k_{1}$ and $k_{2}$; this is best seen when the link is drawn in a symmetric form as in Figure 6. Note that $\Sigma$ is a hyperbolic homology sphere.

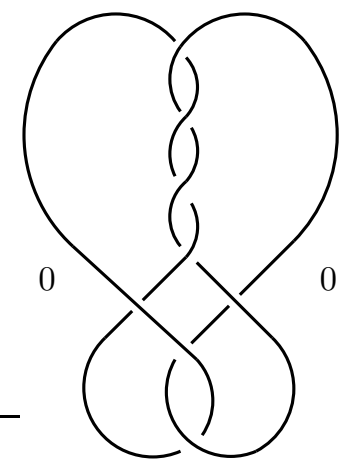

Figure 6

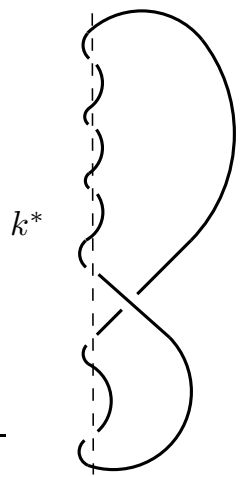

Figure 7

The Floer homology of $\Sigma$ is trivial in even degrees, and is a copy of $\mathbb{Z}$ in each of the odd degrees, see [28]. Therefore, $\tau_{*}: I_{n}(\Sigma) \rightarrow I_{n}(\Sigma)$ is necessarily plus or minus identity for each $n$. It turns out that the exact answer can be obtained from computing the Lefschetz number of $\tau_{*}$ keeping in mind that condition $(*)$ of Section 3.7 is satisfied because the character variety $\mathcal{R}^{*}(\Sigma)$ is non-degenerate, see 28 .

The manifold $\Sigma / \tau$ is obtained from $S^{3}$ by surgery on the knot $k^{*}$ which is the image of the link $k_{1} \cup k_{2}$, see Figure 7 . Notice that the canonical longitudes of $k_{1}$ and $k_{2}$ project onto a longitude of $k^{*}$ whose linking number with $k^{*}$ equals one. The dotted line in Figure 7 represents the branch set $k$. 
This picture can be viewed as a surgery description of the knot $k$ in $\Sigma / \tau=S^{3}$. A little exercise in Kirby calculus shows that $k$ is obtained from the left-handed $(5,6)$-torus knot on six strings by adding one full left-handed twist on two adjacent strings. The signature of $k$ can differ by at most two from the signature of the left-handed $(5,6)$-torus knot, which equals 16 . Since sign $k$ must be divisible by eight, we conclude that $\operatorname{sign} k=16$. Therefore the Lefschetz number of $\tau_{*}$ equals 2 . This implies that $\tau_{*}=-\mathrm{id}: I_{*}(\Sigma) \rightarrow I_{*}(\Sigma)$.

\section{References}

[1] Selman Akbulut, A fake compact contractible 4-manifold, J. Differential Geom. 33 (1991) 335-356

[2] Hans U Boden, Andrew Nicas, Universal formulae for SU(n) Casson invariants of knots, Trans. Amer. Math. Soc. 352 (2000) 3149-3187

[3] Steven Boyer, Andrew Nicas, Varieties of group representations and Casson's invariant for rational homology 3-spheres, Trans. Amer. Math. Soc. 322 (1990) 507-522

[4] Steven Boyer, Daniel Lines, Surgery formulae for Casson's invariant and extensions to homology lens spaces, J. Reine Angew. Math. 405 (1990) 181-220

[5] Peter J Braam, Simon K Donaldson, Floer's work on instanton homology, knots and surgery, from: "The Floer memorial volume", Progr. Math. 133, Birkhäuser, Basel (1995) 195-256

[6] Peter J Braam, Gordana Matić, The Smith conjecture in dimension four and equivariant gauge theory, Forum Math. 5 (1993) 299-311

[7] Olivier Collin, Nikolai Saveliev, Equivariant Casson invariants via gauge theory, J. Reine Angew. Math. 541 (2001) 143-169

[8] Simon K Donaldson, The orientation of Yang-Mills moduli spaces and 4manifold topology, J. Differential Geom. 26 (1987) 397-428

[9] Simon K Donaldson, Floer Homology Groups in Yang-Mills Theory, Cambridge University Press (2002)

[10] Ronald Fintushel, Ronald J Stern, Instanton homology of Seifert fibred homology three spheres, Proc. London Math. Soc. 61 (1990) 109-137

[11] Ralph H Fox, Knots and periodic transformations, from: "Topology of 3manifolds and related topics (Proc. The Univ. of Georgia Institute, 1961)", Prentice-Hall, Englewood Cliffs, NJ (1962) 177-182

[12] Charles Frohman, Andrew Nicas, An intersection homology invariant for knots in a rational homology 3-sphere, Topology 33 (1994) 123-158

[13] Mikio Furuta, Hiroshi Ohta, Differentiable structures on punctured 4manifolds, Topology Appl. 51 (1993) 291-301 
[14] Robert E Gompf, András I Stipsicz, 4 -manifolds and Kirby calculus, American Mathematical Society, Providence, RI (1999)

[15] Christopher M Herald, Flat connections, the Alexander invariant, and Casson's invariant, Comm. Anal. Geom. 5 (1997) 93-120

[16] Christopher M Herald, Legendrian cobordism and Chern-Simons theory on 3-manifolds with boundary, Comm. Anal. Geom. 2 (1994) 337-413

[17] Christopher M Herald, Transversality for equivariant gradient systems and gauge theory on 3-manifolds (2003), preprint

[18] Jerome P Levine, Polynomial invariants of knots of codimension two, Ann. of Math. (2) 84 (1966) 537-554

[19] Kaneko Masataka, Casson's knot invariant and gauge theory, Topology Appl. 112 (2001) 111-135

[20] Walter D Neumann, Jonathan Wahl, Casson invariant of links of singularities, Comment. Math. Helv. 65 (1990) 58-78

[21] Walter D Neumann, An invariant of plumbed homology spheres, from: "Topology Symposium, Siegen 1979 (Proc. Sympos., Univ. Siegen, Siegen, 1979)", Lecture Notes in Math. 788, Springer, Berlin (1980) 125-144

[22] Liviu I Nicolaescu, Notes on Seiberg-Witten theory, American Mathematical Society, Providence, RI (2000)

[23] Daniel Ruberman, Doubly slice knots and the Casson-Gordon invariants, Trans. Amer. Math. Soc. 279 (1983) 569-588

[24] Daniel Ruberman, Nikolai Saveliev, Rohlin's invariant and gauge theory I. Homology 3-tori (2003), Comm. Math. Helv. (to appear). http://front.math.ucdavis.edu/math.GT/0302131

[25] Dietmar A Salamon, Seiberg-Witten invariants of mapping tori, symplectic fixed points, and Lefschetz numbers, from: "Proceedings of 6th Gökova Geometry-Topology Conference", Turkish J. Math. 23 (1999) 117-143

[26] Nikolai Saveliev, Floer homology of Brieskorn homology spheres, J. Differential Geom. 53 (1999) 15-87

[27] Nikolai Saveliev, Representation spaces of Seifert fibered homology spheres, Topology Appl. 126 (2002) 49-61

[28] Nikolai Saveliev, A note on Akbulut corks, Math. Res. Lett. 10 (2003) 777-786

[29] Laurent Siebenmann, On vanishing of the Rohlin invariant and non-finitely amphicheiral homology 3-spheres, from: "Topology Symposium, Siegen 1979 (Proc. Sympos., Univ. Siegen, Siegen, 1979)", Lecture Notes in Math. 788, Springer, Berlin (1980) 172-222

[30] Clifford H Taubes, Casson's invariant and gauge theory, J. Differential Geom. 31 (1990) 547-599

[31] Shuguang Wang, Moduli spaces over manifolds with involutions, Math. Ann. 296 (1993) 119-138 